\title{
Resultados sobre Metaestabilidade num Modelo de Ising Estocástico
}

\section{Eduardo Jordão Neves}

Tese apresentada

ao

Instituto de Matemática e Estatística

da

Universidade de São Paulo

para obtenção do grau de Doutor

em

Estatística

Área de Concentração: Probabilidades

Orientador: Prof. Dr. Roberto Henrique Schonmann

-São Paulo, Junho de 1990- 


\section{Agradecimentos}

Várias pessoas contribuiram de diversas formas neste trabalho. Em especial gostaria de agradecer

- ao Roberto, pela amizade e por tudo que me ensinou.

- ao Galves, pela iniciação em teoria de probabilidades e, em particular, ao problema da metaestabilidade.

- a Beti, pela amizade (e pelas dicas no $\mathrm{L}_{\mathrm{E}} \mathrm{X}$ ).

- ao Perez, Wreszinski, Pablo, Nelson e João, pelo estímulo e proveitosas discussões.

- ao Walter Vicente Fernandes, pelo auxílio na datilografia deste trabalho. 


\section{ÍNDICE}

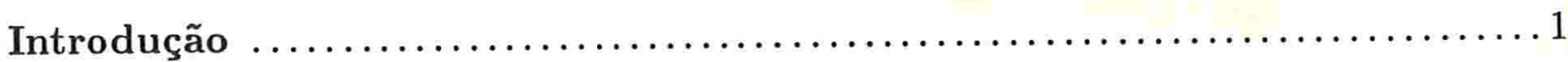

Capítulo 1 Estrutura de energia no espaço de configurações e tempos de

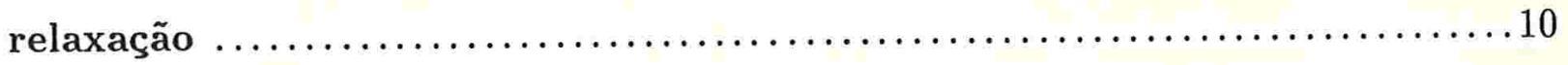

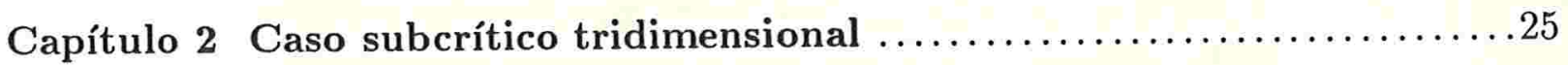

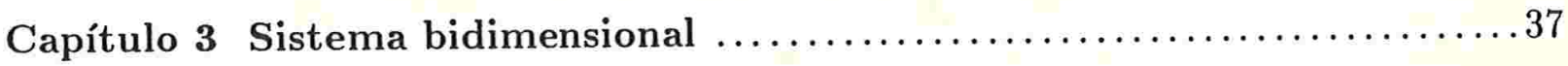

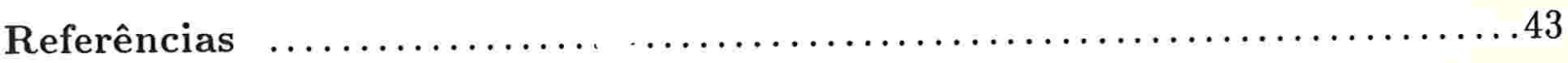




\section{Abstract}

We consider some aspects concerning the metastable behavior of the Ising Model with the so called Metropolis dynamics for both volume and magnetic field held fixed while the temperature goes to zero. Results on the stability of small droplets of spin +1 in a sea of -1 spins in dimension three are obtained through an analysis of the energy structure in the configuration space and the resulting behavior of the process. This analysis also yelds results in the two dimensional case already presented in [NS]. 


\section{Resumo}

Consideramos alguns aspectos do comportamento metaestável do modelo de Ising dotado da chamada dinâmica de Metrópolis em dimensão dois e três para volume e campo magnético positivo mantidos fixos no limite no qual a temperatura vai a zero. Resultados sobre a estabilidade de blocos pequenos de spin +1 imersos num mar de spins -1 em dimensão três são obtidos através da análise da estrutura de energia no espaço de configurações e do comportamento do processo frente a ela. Esta análise também permite obter vários resultados para o sistema bidimensional já apresentados em [NS]. 


\section{Introdução}

Neste trabalho estudamos alguns aspectos do comportamento metaestável do processo estocástico definido pelo modelo de Ising ferromagnético dotado de uma dinâmica de Glauber no limite de baixas temperaturas no volume finito. A caracterização de comportamento metaestável aqui adotada é aquela que considera a evolução ao longo de trajetórias, o chamado "pathwise approach" ou abordagem trajetória por trajetória, introduzido em [CGOV] e posteriormente empregada e desenvolvida em vários contextos em $[\mathrm{KN}],[\mathrm{Sch}],[\mathrm{NCK}],[\mathrm{GOV}],[\mathrm{COP}],[\mathrm{MOS}]$, [EGJL] e [Bra]. Nesta abordagem a metaestabilidade se manifestaria no comportamento de trajetórias típicas do processo em questão. Para escolhas convenientes do estado inicial a evolução do processo aparenta ter atingido o equilíbrio no qual permanece durante um tempo bastante longo até que, num instante imprevisível e de forma brusca, passa ao verdadeiro estado de equilíbrio. A análise da evolução temporal de distribuições de probabilidade associadas ao processo poderia não detétar esse comportamento devido às flutuações do tempo de transição serem muito grandes.

$\mathrm{O}$ modelo de Ising ferromagnético tem Hamiltoniano dado por

$$
H(\eta)=-\frac{1}{2} \sum_{\langle x, y\rangle} \eta(x) \eta(y)-\frac{h}{2} \sum_{x} \eta(x)
$$

onde $x, y \in \Lambda_{N}:=\{1,2, \ldots, N\}^{d}, d$ sendo a dimensão da rede subjacente, com condições periódicas na fronteira, $\eta \in \Sigma:=\{-1,+1\}^{\Lambda_{N}}$ é uma configuração na qual $\eta(x)$ é o valor do spin no ponto $x$; o primeiro termo representa a soma sobre os pares de vizinhos próximos $\mathrm{e}$ 
o segundo representa a soma sobre os sítios do toro d-dimensional. O termo $h$ corresponde ao campo magnético que tomamos não negativo.

Através desse Hamiltoniano definimos um processo estocástico Markoviano a tempo contínuo $\left\{\sigma_{t}^{\eta}\right\}_{t \geq 0}$ em $\Sigma$ satisfazendo:

$$
\begin{aligned}
& P\left(\sigma_{0}^{\eta}=\eta\right)=1 \text { e se } \zeta, \rho \in \Sigma, \zeta \neq \rho \text { então } \\
& P\left(\sigma_{t+\epsilon}^{\eta}=\zeta \mid \sigma_{t}^{\eta}=\rho\right)= \begin{cases}c(x, \rho) \epsilon+o(\epsilon) & \text { se } \zeta=\rho^{x} \text { para algum } x \\
o(\epsilon) & \text { caso contrário }\end{cases}
\end{aligned}
$$

onde

$$
\rho^{x}(y)= \begin{cases}\rho(y) & \text { se } x \neq y \\ -\rho(y) & \text { caso contrário }\end{cases}
$$

e $c(x, \eta) \geq 0$ é a taxa com a qual o spin no ponto $x$ vira quando a configuração é $\rho$.

Fazemos ainda a restrição de que esse processo seja reversível com respeito a uma particular medida em $\Sigma$ : a medida de Gibbs à temperatura inversa $\beta \geq 0$, definida por

$$
\mu(\eta)=\frac{e^{-\beta H(\eta)}}{\sum_{\zeta \in \Sigma} e^{-\beta H(\zeta)}}
$$

onde a condição de reversibilidade é dada por

$$
\mu(\eta) c(x, \eta)=\mu\left(\eta^{x}\right) c\left(x, \eta^{x}\right)
$$

Estas condições dotam o modelo de Ising com a chamada dinâmica de Glauber [Gla]. O processo estocástico assim definido é chamado de modelo de Ising estocástico em [Lig].

Naturalmente existem infinitas taxas satisfazendo (0.5) mas vamos nos restringir a chamada dinâmica de Metrópolis dada por

$$
c(x, \eta)= \begin{cases}e^{-\beta \Delta_{x} H(\eta)} & \text { se } \Delta_{x} H(\eta)>0 \\ 1 & \text { caso contrário }\end{cases}
$$

onde $\Delta_{x} H(\eta)=H\left(\eta^{x}\right)-H(\eta)$. 
Com essas taxas o processo pode ser construído escolhendo aleatoriamente, com taxa $N^{d}$, um sítio de $\Lambda_{N}$ e virando o spin desse sítio com probabilidade $c(x, \eta)$ onde $\eta$ é a presente configuração.

Para $\eta \in \Sigma$ e $A \subset \Sigma$ definimos

$$
T^{\eta}(A)=\inf \left\{t \geq 0: \sigma_{t}^{\eta} \in A\right\}
$$

sendo que se $A=\{\zeta\}$ escrevemos apenas $T^{\eta}(\zeta)$. Convencionamos também que quando $\eta=-1$ ele será omitido, onde -1 corresponde à configuração na qual todos os spins têm valor -1 , e se $A=\{+\underline{1}\}$, onde $+\underline{1}$ corresponde à configuração na qual todos os spins tem valor $+1, \mathrm{~A}$ também será omitido.

Quando $\beta$ cresce, com $N$ e $h>0$ fixos, $\mu$ concentra sua massa em +1 . No entanto se o campo magnético não for muito intenso o estado $-\underline{1}$ será um mínimo local de energia se $0<\beta<\infty$. Existem, de fato, vários mínimos locais de energia de diferentes magnitudes no espaço de configurações. Neste trabalho vamos apresentar alguns resultados sobre o comportamento metaestável do processo frente a essa estrutura de mínimos e barreiras de energia no limite de baixas temperaturas para sistemas em dimensão dois e três.

O sistema unidimensional é relativamente simples e além de informação sobre a dinâmica do processo podemos também obter resultados interessantes ( [TS], [N] ) sobre o comportamento da medida de equilíbrio desse sistema quando tomamos conjuntamente os limites $N$ e $\beta$ indo para infinito. Omitiremos, no entanto, esses resultados, nos concentrando nos casos bi e tridimensionais.

Uma primeira observação importante é que, em qualquer dimensão, não teremos comportamento metaestável se o campo magnético for suficientemente forte. De fato, seja $\left\{\bar{\sigma}_{t}^{\eta}\right\}_{t \geq 0}$, o processo com configuração inicial $\eta$ no qual cada spin -1 vira com taxa 1 e os spins +1 estão congelados, ou seja, viram com taxa 0 . Então vale

Proposição 1 Se $h>2 d$ e $\eta \in \Sigma$ podemos construir os dois processos $\left\{\sigma_{t}^{\eta}\right\}$ e $\left\{\bar{\sigma}_{t}^{\eta}\right\}$ no 
mesmo espaço de probabilidades tal que, para todo $t_{0}<\infty$

$$
\lim _{\beta \rightarrow \infty} P\left(\sigma_{t}^{\eta} \neq \bar{\sigma}_{t}^{\eta}, \text { para algum } t \in\left[0, t_{0}\right]\right)=0
$$

A situação fica mais complicada e mais interessante quando tomamos o campo magnético pequeno e é essa a situação na qual iremos nos concentrar.

Vamos apresentar agora os resultados principais para o sistema bidimensional ( [NS] ).

Seja $\mathcal{R}$ o conjunto de configurações com todos os spins -1 exceto aqueles no interior de um retângulo $l_{1} \times l_{2}$ com $l_{1}$ e $l_{2}$ menores que $N-1$. Para $\eta \in \mathcal{R}$ defina $l(\eta)=\min \left(l_{1}, l_{2}\right)$.

Teorema 1 Suponha que $d=2,0<h<1$ e que $\eta \in \mathcal{R}$. Então para todo $\epsilon>0$

a) $\operatorname{Se} l(\eta)=l<2 / h$ e $\epsilon>0$ então

$$
\lim _{\beta \rightarrow \infty} P\left(T^{\eta}(-1)<T^{\eta}(+1), T^{\eta}(-\underline{1})<e^{\beta(l-1+\varepsilon)}\right)=1
$$

b) $S \in l(\eta)>2 / h$ então

$$
\lim _{\beta \rightarrow \infty} P\left(T^{\eta}(+\underline{1})<T^{\eta}(-\underline{1}), T^{\eta}(+\underline{1})<e^{\beta(2-h+\epsilon)}\right)=1
$$

Este teorema caracteriza $L:=\lceil 2 / h\rceil=$ menor inteiro maior ou igual a $2 / h$ como o tamanho crítico de uma gota quadrada. Este valor está de acordo com o que se poderia esperar por argumentos heurísticos. Por exemplo o valor $2 / h$ pode ser obtido maximizando a energia de uma configuração quadrada de $\mathcal{R}$ em função do lado. Já de um ponto de vista dinâmico poderíamos admitir que a evolução a partir de uma configuração inicial em $\mathcal{R}$ seria governada pelo resultado da competição entre um processo de erosão e um processo de criação de protuberâncias. No primeiro, spins +1 nos cantos ( ou seja, spins +1 com dois vizinhos de mesmo sinal em direções perpendiculares ) seriam removidos ou recuperados de tal forma que o número total de spins +1 perdidos no interior 
da configuração inicial $\eta \in \mathcal{R}$ teria aproximadamente o mesmo comportamento que um processo de nascimento e morte com taxa de nascimento da ordem de $e^{-\beta h}$ e de morte da ordem de 1. Quando $l(\eta)-1$ cantos são removidos, atingimos, com grande probabilidade para $\beta$ grande, uma configuração que consiste num retângulo de spins +1 com uma fatia externa a menos em relação a $\eta$ junto com um único spin +1 nesta fatia. Como este último spin vira com taxa 1 esta heurística diria que a gota decresce no tempo necessário para que o processo de nascimento e morte atinja o estado $l(\eta)-1$, ou seja, um tempo da ordem de $e^{\beta(l(\eta)-2) h}$. O processo de criação de protuberâncias corresponde ao aparecimento de spins +1 adjacentes a um dos lados de $\eta$. Como a partir dessa configuração " $\eta+$ protuberância" podemos atingir uma configuração maior através de transições com taxa 1 , esse processo ocasionaria o crescimento da gota num tempo da ordem de $e^{\beta(2-h)}$. O ponto crítico seria aquele no qual houvesse empate entre essas duas tendências o que ocorre para $l(\eta)=2 / h$.

Uma demonstração no sentido de tornar rigorosa essa heurística é encontrada em [NS]. A demonstração desse teorema que aqui apresentamos explora a estrutura de ener ergia do espaço de configurações bidimensional que é bastante simples. Cada configuração de $\eta \in \mathcal{R}$ corresponde a um mínimo local de energia e definirá uma bacia local de atração que denominaremos de cone de configurações de $\eta$. Para $\beta$ grande o processo partindo de $\eta$ passa a maior parte do tempo próximo ao fundo do cone e, para atingir um outro mínimo local de energia, precisa vencer uma barreira de energia a qual, naturalmente, vai depender de $\eta$. No caso bidimensional vão existir três tipos de mínimos de energia. Se a barreira energética for menor ou igual a $(L-2) h$ então vale a) do Teorema 1 . Se for igual a $2-h$ então vale b). A outra possibilidade, correspondendo às duas configurações $+\underline{1} \mathrm{e}$ -1 , tem barreira energética superior a $2-h$.

A estrutura de energia para o caso tridimensional, como veremos, é bem mais elaborada.

Vamos impor agora algumas restrições ao sistema considerado tanto no caso bidimensional quanto no caso tridimensional. Diremos que estamos no caso fundamental em d dimensões se :0<h<1 com $h$ tal que $2 / h$ não é um número inteiro e $N>N(h):=$ $2 L^{d}$. 
A primeira restrição contida na definição do caso fundamental considera o caso mais complicado e mais interessante no qual o campo é pequeno. A segunda vem do fato de se $2 / h$ for um número inteiro surgirem certas particularidades que, sem apresentarem dificuldades essenciais, dificultam a exposição. Uma vez de posse dos resultados para o sistema com essa restrição é relativamente fácil estendê-los para o caso geral. A última restrição é feita para que a passagem do sistema do estado $-\underline{1}$ ao estado $+\underline{1}$ seja através da formação de uma gota crítica e não pela formação de um anel de spins +1 ao redor do toro.

O próximo resultado exibe o caráter imprevisível do instante da passagem do estado $-\underline{1}$ ao estado $+\underline{1}$.

Teorema 2 Parad $=2$ no caso fundamental

$$
\frac{T}{E T} \longrightarrow \tau \text { em distribuição quando } \beta \rightarrow \infty
$$

onde $\tau$ é uma variável aleatória exponencial com média 1 e $T:=T^{-1}(+\underline{1})$.

O próximo teorema fornece alguma informação sobre a forma com que o processo passa de -1 a +1 e sobre a magnitude do tempo gasto nesta transição. Antes de enunciá-lo precisamos de algumas definições.

Seja $\mathcal{G}$ o conjunto das configurações de $\mathcal{R}$ nas quais os spins +1 formam uma gota quadrada de lado L. Também definimos $\mathcal{P}$ como o conjunto das configurações nas quais todos os spins são -1 exceto aqueles no interior de um retângulo $L \times(L-1)$ juntamente com um spin +1 adjacente a um dos lados maiores. Pelo Teorema $1, \mathcal{G}$ é o conjunto das gotas criticas enquanto $\mathcal{P}$ seria o conjunto de configurações que poderíamos chamar de proto-gotas críticas uma vez que uma configuração de $\mathcal{P}$ pode, com probabilidades que não vão a zero quando $\beta$ cresce, tanto crescer para uma gota crítica quanto diminuir a um retângulo $L \times(L-1)$ que, pelo Teorema 1 , é subcrítico.

Para $\eta \in \mathcal{P}$ defina

$$
\Gamma(h):=H(\eta)-H(-1)=4 L-L^{2} h+(L-1) h
$$




\section{Teorema 3 Parad $=2$ no caso fundamental}

a)

$$
\lim _{\beta \rightarrow \infty} \frac{1}{\beta} \log T=\Gamma(h) \text { em probabilidade }
$$

b)

$$
\lim _{\beta \rightarrow \infty} \frac{1}{\beta} \log E T=\Gamma(h)
$$

c)

$$
\lim _{\beta \rightarrow \infty} P(T(\mathcal{G})<T)=1
$$

d)

$$
\lim _{\beta \rightarrow \infty} P(T(\mathcal{P})<T)=1
$$

Vamos agora considerar o caso tridimensional. O resultado que apresentamos corresponde ao análogo em três dimensões da parte a) do Teorema 1.

Primeiro verifiquemos qual, heuristicamente, deve ser o tamanho crítico de uma gota tridimensional tal que gotas maiores tendam a crescer e gotas menores tendâm a decrescer.

Suponha que $\eta \in \Sigma$ é uma configuração na qual todos os spins são -1 exceto aqueles no interior de um paralelepípedo de lados $\mathrm{P}, \mathrm{Q}$ e R. Suponha $P \leq Q \leq R$.

Como no caso bidimensional, podemos considerar o máximo de energia de uma configuração cúbica em função de seu lado $l$. Nesse caso temos $H(\eta)-H(-1)=6 l^{2}-l^{3} h$ cujo máximo é assumido para $l=4 / h$.

De um ponto de vista dinâmico poderíamos, de novo como no caso bidimensional, procurar a caracterização da gota crítica através da análise da competição entre os processos de erosão e de crescimento. Agora o processo de erosão precisaria eliminar o suficiente de uma face externa do paralepípedo para que o que restasse tivesse tendência a diminuir e não a recuperar essa face. Como sobre uma face do paralepípedo a dinâmica é 
de certa forma bidimensional, a heurística é que é necessário que não sobre mais que uma proto-gota crítica bidimensional nessa face. Para o processo de crescimento a situação é análoga. Para que a gota cresça seria necessário o aparecimento de uma protuberância suficientemente grande em uma das faces a qual, por sua vez, tenha tendência a crescer e preencher uma fatia extra ao paralelepípedo original. Essa protuberância deveria ser, então, maior ou igual à proto-gota crítica bidimensional.

O que nos impede de prosseguir esse argumento em analogia com caso bidimensional é que tanto o processo de erosão quanto o de crescimento são, agora, bastante complicados. Podemos observar, no entanto, que a dificuldade em atingir uma configuração cresce conforme aumenta a diferença entre as energias da configuração final e a da final e, portanto, cresce o tempo necessário para que isso ocorra. Nesse sentido a caracterização da gota crítica seria obtida comparando o custo de energia necessário para acrescentar uma proto-gota crítica numa face com aquela necessária para erodir uma face do paraálelepípedo deixando sobre ela apenas uma proto-gota crítica. Primeiro observe que se uma das faces do paralelepípedo for, ela mesma subcrítica, o que ocorre se $P<L$, então a tendência é decrescer.
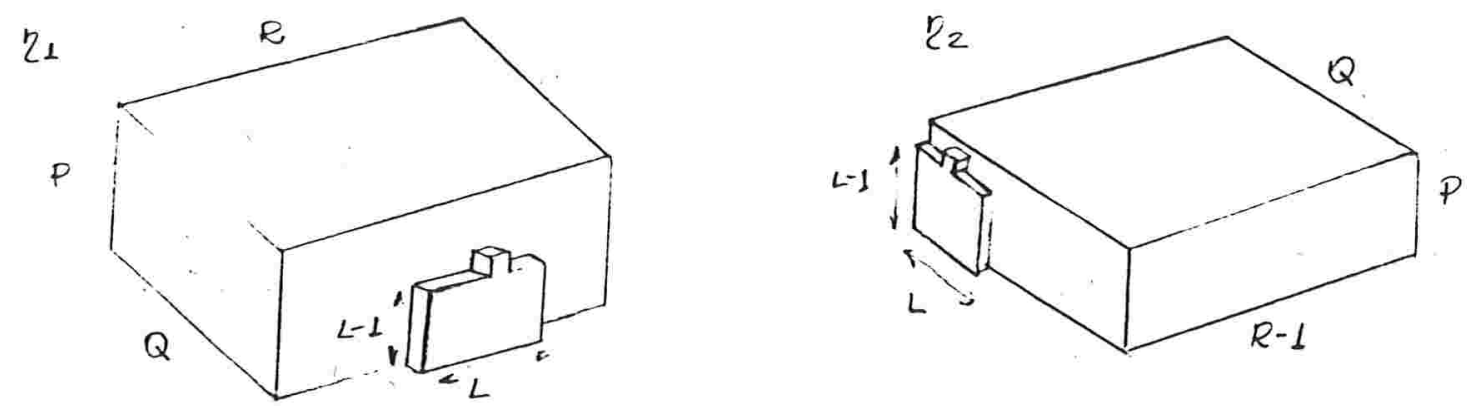

Se $\eta_{1}$ é uma configuração obtida de $\eta$ pelo acréscimo de uma proto-gota crítica em uma face e $\eta_{2}$ é uma configuração obtida de $\eta$ removendo uma das faces externas de menor área ( i.e., uma daquelas de lados P e Q ) e sobre esta adicionando uma proto-gota crítica, então

$$
H\left(\eta_{1}\right)-H\left(\eta_{2}\right)=2(P+Q)-P Q h
$$

o que fornece o valor crítico $Q_{c}=\frac{2 P}{P h-2}$.

Note que caso $P=Q=R=l$ recuperamos o valor crítico $4 / h$. 
No capítulo 2 mostraremos que de fato a região indicada na figura é subcrítica ou mais precisamente

Teorema 4 Seja $\eta \in \mathcal{R}$ com $P<L$ ou $P \geq L$ e $Q<Q_{Q}$ no caso fundamental. Então, se $\epsilon>0$

$$
\lim _{\beta \rightarrow \infty} P\left(T^{\eta}(-\underline{1})<T^{\eta}(+\underline{1}), T^{\eta}(-\underline{1})<e^{\beta(E(h)+\epsilon)}\right)=1
$$

onde $E(h)=\Gamma(h)-2(P+Q)+P Q h$.

Observe que $E(h)$ é exatamente o custo energético para se alcançar $\eta_{2}$ a partir de $\eta$.

Acreditamos que o caso supercrítico também possa ser tratado utilizando as técnicas aqui desenvolvidas e temos a mesma expectativa com relação aos análogos dos Teoremas 2 e 3 para o caso tridimensional. Deixamos, no entanto, estes problemas para uma futura investigação.

Estes resultados são apresentados em três capítulos.

Capítulo 1: apresentamos os resultados básicos que serão usados nos demais capítulos. Basicamente eles descrevem a estrutura de mínimos e de barreiras de energia no espaço de configurações em dimensão geral e seu efeito na evolução do processo para diversas escalas de tempo.

Capítulo 2: demoŝtramos o Teorema 4 aplicando os resultados do capítulo 1 no caso tridimensional subcrítico.

Capítulo 3: demonstramos os Teoremas 1 e 2 e apresentamos o esquema da demonstração do Teorema 3. 


\section{Capítulo 1}

\section{Estrutura de energia no espaço de configurações e tempos de relaxação.}

Vamos aqui determinar a estrutura de mínimos locais de energia no espaço de configurações junto com os resultados que esta origina e que serão usados nos capítulos seguintes.

Notação: Dadas duas configurações $\eta$ e $\zeta$, escrevemos $\langle\eta, \zeta\rangle$ se existe um $x \in \Lambda_{N}$ tal que $\eta^{x}=\zeta$, ou seja, se é possível passar de uma à outra através de uma transição do processo.

Uma observação importante é que, devido às restrições quanto ao campo $h$ contidas na definição de caso fundamental, temos sempre $H(\eta)-H\left(\eta^{x}\right) \neq 0$.

A definição de mínimos mais local que podemos fazer é: 


\section{Definição:}

$$
M=\{\eta: \forall \zeta \text { com }\langle\eta, \zeta>\text { então } H(\zeta)>H(\eta)\}
$$

Qualquer configuração "relaxa num tempo de ordem 1 para uma configuração de $M$ " ou, mais precisamente:

\section{Lema 1 Para todo $\xi \in \Sigma$ e $\epsilon>0$}

$$
P\left(\sigma_{t}^{\xi} \in M, \text { para algum } t<e^{\beta \epsilon}\right) \rightarrow 1 \text { quando } \beta \rightarrow \infty
$$

Dem : Basta considerar $\xi \in \Sigma \backslash M$. Neste caso, com probabilidade tendendo a 1 quando $\beta$ cresce, a primeira transição leva a uma configuração $\xi_{1} \operatorname{com} H\left(\xi_{1}\right)<H(\xi)$. Se $\xi_{1} \in \Sigma \backslash M$ o mesmo argumento se repete gerando uma sequência de configurações $\xi_{1}, \xi_{2}, \ldots$ com $<\xi_{i}, \xi_{i+1}>$, e $H\left(\xi_{i+1}\right)<H\left(\xi_{i}\right)$. Como $|\Sigma|=2^{N^{d}}<\infty$ essa sequência termina, ou seja, uma configuração em $M$ é alcançada. Como a limitação no tamanho da sequência não depende de $\beta$ e as transições que aumentam a energia têm taxa não superior a $e^{-\beta h}$, segue o lema.

$\square_{\text {Lema } 1}$

Para cada $\eta \in M$ definimos seu cone de configurações, $C(\eta)$, como o conjunto das configurações que têm probabilidade positiva, uniformemente em $\beta$, de atingir $\eta$ antes de qualquer outra configuração de $M$. Definimos também a fronteira do cone de $\eta, \partial C(\eta)$, como o subconjunto das configurações de $C(\eta)$ que podem deixá-lo em uma transição, que necessariamente vai diminuir a energia, e a borda do cone, $\bar{C}(\eta)$, como o subconjunto das configurações da fronteira com energia mínima, a qual será escrita como $H(\eta)+\delta_{\eta}$ de forma que $\delta_{\eta}$ corresponde à altura da barreira energética de $C(\eta)$. Mais precisamente, se $\eta \in M$, definimos

$$
C(\eta):=\left\{\xi \in \Sigma: \text { existe uma sequência } \xi_{0}=\eta, \xi_{1}, \ldots, \xi_{k}=\xi\right.
$$

para algum $k \in I N$, com $<\xi_{i}, \xi_{i+1}>$ e $\left.H\left(\xi_{i}\right)<H\left(\xi_{i+1}\right), i=1,2, \ldots, k-1\right\}$ 


$$
\begin{gathered}
\partial C(\eta):=\{\xi \in C(\eta) \cap C(\rho) \text { para } \rho \neq \eta \text { e } \rho \in M\} \\
\delta_{\eta}:=\min \{H(\xi)-H(\eta), \xi \in \partial C(\eta)\} \\
\bar{C}(\eta):=\left\{\xi \in \partial C(\eta): H(\xi)=H(\eta)+\delta_{\eta}\right\}
\end{gathered}
$$

Observe que $\Sigma=\bigcup_{\eta \in M} C(\eta)$.

Uma técnica bastante utilizada neste trabalho será a de acoplar o processo original com um processo restrito à uma certa região conexa $\mathcal{S}$. Dizemos que $\mathcal{S}$ é conexo se para todo par $\xi$ e $\rho$ de configurações em $\mathcal{S}$ existe uma sequência $\xi_{0}=\xi, \xi_{1}, \ldots, \xi_{k}=\rho$, para algum $k$, com $<\xi_{i}, \xi_{i+1}>$ e $\xi_{i} \in \mathcal{S}$ para $i=1,2, \ldots, k-1$.

Serão usados basicamente dois acoplamentos que, como em [NS], chamaremos de acoplamentos A e B que passamos a definir. Seja $\left\{\tilde{\sigma}_{t}^{\eta}\right\}_{t \geq 0}$ o processo com configuração inicial $\eta \in \mathcal{S}, \mathcal{S}$ um conjunto conexo, obtido a partir do processo original, $\left\{\sigma_{t}^{\eta}\right\}_{t \geq 0}$, cancelando as taxas correspondentes a transições entre configurações de $\mathcal{S}$ e $\mathcal{S}^{c}$.

Acoplamento A: O processo original, $\left\{\sigma_{t}^{\eta}\right\}_{t \geq 0}$, e o processo restrito a $\mathcal{S},\left\{\tilde{\sigma}_{t}^{\eta}\right\}_{t \geq 0}$ evoluem juntos até que o primeiro escape de $\mathcal{S}$; neste instante o processo restrito fica parado e a partir daí os dois evoluem independentemente.

Acoplamento B: Se $\xi \in \mathcal{S}$ os processos $\left\{\tilde{\sigma}_{t}^{\eta}\right\}_{t \geq 0}$ e $\left\{\tilde{\sigma}_{t}^{\xi}\right\}_{t \geq 0}$ evoluem independentemente até que se encontrem; a partir desse instante eles evoluem juntos.

Em algumas aplicaçôes do acoplamento B a configuração inicial $\xi$ será escolhida conforme a medida $\tilde{\mu}$ definida por

$$
\tilde{\mu}(\eta)= \begin{cases}\frac{\mu(\eta)}{\sum_{\rho \in S}^{\mu(\rho)}} & \text { se } \rho \in \mathcal{S} \\ 0 & \text { caso contrário }\end{cases}
$$


onde $\mu$ é a medida de Gibbs associada ao modelo. Em cada aplicação teremos um conjunto $\mathcal{S}$ mas, por simplicidade, usamos sempre a mesma notação, $\tilde{\mu}$, para essa medida restrita. Da mesma forma usamos sempre a notação $\left\{\tilde{\sigma}_{\imath}^{\eta}\right\}_{t \geq 0}$ para o processo restrito, naturalmente $\operatorname{com} \eta$, a configuração inicial, pertencendo ao particular conjunto $\mathcal{S}$ em questão.

Vamos verificar agora que, com probabilidade tendendo a 1 quando $\beta$ cresce, o processo sai de um cone através de uma configuração de sua borda e determinaremos a magnitude do tempo de saída. Para isso precisaremos do seguinte resultado que fornece uma limitação inferior para o tempo até o processo atingir uma dada configuração.

Lema 2 Sejam $\mathcal{S}$ conexo e $\eta \in \mathcal{S}$ tal que para todo $\xi \in \mathcal{S} \backslash\{\eta\}$ vale $H(\xi)>H(\eta)$. Então para todo $\xi \in \mathcal{S}$ e $\epsilon>0$

$$
\lim _{\beta \rightarrow \infty} P\left(\tilde{T}(\xi)<e^{\beta(H(\xi)-H(\eta)-\epsilon)}\right)=0
$$

onde $\tilde{T}(\xi)=\inf \left\{t \geq 0: \tilde{\sigma}_{t}^{\eta}=\xi\right\}$

Dem: $O$ argumento vem de [LS]. Acoplamos $\left\{\tilde{\sigma}_{t}^{\eta}\right\}$ e $\left\{\tilde{\sigma}_{t}^{\tilde{\mu}}\right\}$ pelo acoplamento B. Então se $\tilde{T}(\xi)=\inf \left\{t \geq 0: \tilde{\sigma}_{t}^{\bar{\mu}}=\xi\right\}$

$$
\begin{aligned}
& P(\tilde{T}(\xi)<\exp (\beta(H(\xi)-H(\eta)-\epsilon))) \leq P\left(\tilde{\sigma}_{0}^{\eta} \neq \tilde{\sigma}_{0}^{\tilde{\mu}}\right)+ \\
& P\left(\tilde{\sigma}_{t}^{\tilde{\mu}}=\xi \text { para algum } t \in\left\{\beta^{-1}, 2 \beta^{-1}, \ldots,[\beta \exp (\beta(H(\xi)-H(\eta)-\epsilon))] \beta^{-1}\right\}\right)+ \\
& P\left(\tilde{\sigma}_{t}^{\tilde{\mu}} \text { muda de estado entre os tempos } \tilde{T}^{\tilde{\mu}}(\xi) \text { e } \tilde{T}^{\tilde{\mu}}(\xi)+\beta^{-1}\right) \leq \\
& \tilde{\mu}(\eta)+(\beta \exp (\beta(H(\xi)-H(\eta)-\epsilon))+1) \tilde{\mu}(\xi)+ \\
& \left(1-\exp \left(-\beta^{-1} N^{d}\right)\right)
\end{aligned}
$$

que vai a zero quando $\beta \rightarrow \infty$.

A magnitude do tempo de saída do cone de uma configuração é dada pelo lema seguinte. 
Lema 3 Se $\eta \in M$

$$
\lim _{\beta \rightarrow \infty} \frac{1}{\beta} \log T^{\eta}(M \backslash\{\eta\})=\delta_{\eta} \text { em probabilidade }
$$

onde $T^{\eta}(B)=\inf \left\{t \geq 0: \sigma_{t}^{\eta} \in B\right\}$ para $B \subset \Sigma$.

Dem : Pelo Lema 1 basta verificar que

$$
\lim _{\beta \rightarrow \infty} \frac{1}{\beta} \log T^{\eta}\left(C^{c}(\eta)\right)=\delta_{\eta} \text { em probabilidade }
$$

Primeiro observe que

$$
P\left(T^{\eta}\left(C^{c}(\eta)\right)>T^{\eta}(\partial C(\eta))\right)=1
$$

e que se tomamos $\mathcal{S}=C(\eta)$ temos

$$
P\left(T^{\eta}(\partial C(\eta))=\tilde{T}^{\eta}(\partial C(\eta))\right)=1
$$

onde $\left\{\sigma_{t}^{\eta}\right\}$ e $\left\{\tilde{\sigma}_{t}^{\eta}\right\}$ tem acoplamento A. Então

$$
\begin{aligned}
& P\left(T^{\eta}\left(C^{c}(\eta)\right)<\exp \left(\beta\left(\delta_{\eta}-\epsilon\right)\right)\right) \leq \\
& P\left(T^{\eta}(\partial C(\eta))<\exp \left(\beta\left(\delta_{\eta}-\epsilon\right)\right)\right)=P\left(\tilde{T}^{\eta}(\partial C(\eta))<\exp \left(\beta\left(\delta_{\eta}-\epsilon\right)\right)\right) \leq \\
& \sum_{\rho \in \partial C(\eta)} P\left(\tilde{T}^{\eta}(\rho)<\exp \left(\beta\left(\delta_{\eta}-\epsilon\right)\right)\right)
\end{aligned}
$$

que vai a zero pelo Lema 2.

Resta obter a limitação superior. Primeiro verificamos que para todo $\eta \in C(\eta)$

$$
P\left(\tilde{T}^{\xi}(\bar{C}(\eta))<1\right) \geq C e^{-\beta \delta_{\eta}}
$$

onde $C$ é uma constante independente de $\beta$.

Para mostrar (1.4) consideremos a seguinte construção do processo, já mencionada na introdução: a cada instante de um processo de Poisson $\{N(t)\}_{t \geq 0}$, com taxa 
$\lambda=N^{d}$, escolhemos um sítio com probabilidade $1 / N^{d}$ e efetuamos a troca do spin desse sítio com probabilidade $c(x, \rho)$ onde $\rho$ é a configuração atual.

Considere uma sequência $\xi_{0}=\xi, \xi_{1}, \ldots, \xi_{k}=\eta, \xi_{k+1}, \ldots, \xi_{k+p} \in \bar{C}(\eta)$ com $<\xi_{i}, \xi_{i+1}>, i=1,2, \ldots, k+p$ para $k, p \in \mathbb{N}$ com energias decrescentes até $\eta$ e em seguida crescentes até $\xi_{k+1}$. Seja $\Omega$ o evento : "o processo $\sigma_{i}^{\xi}$ evolue conforme a sequência $\left\{\xi_{i}\right\}_{i=0}^{k+p}$ atingindo $\bar{C}(\eta)$ antes do instante 1 ". Então

$$
P\left(\tilde{T}^{\eta}(\bar{C}(\eta))<1\right) \geq P(\Omega) \geq\left(\frac{1}{N^{d}}\right)^{k+p} P(N(1) \geq k+p) \prod_{i=0}^{k+p-1} c\left(x_{i}, \xi_{i}\right)
$$

onde $x_{i}$ é o sítio que vira na transição de $\xi_{i}$ para $\xi_{i+1}$. Mas

$$
P(N(1) \geq k+p)>C_{1}>0
$$

independentemente de $\beta$ e

$$
\prod_{i=0}^{k+p-1} c\left(x_{i}, \xi_{i}\right)=e^{-\beta \delta_{\eta}}
$$

De forma que (1.4) segue com

$$
C=C_{1}\left(\frac{1}{N^{d}}\right)^{k+p}
$$

No lema seguinte mostramos que a saida, com probabilidade tendendo a 1 quando $\beta$ cresce, ocorre por $\bar{C}(\eta)$. Antes precisamos de algumas definições.

Para $\eta \in M$ definimos os tempos de parada

$$
\begin{aligned}
\pi_{1}^{\eta} & =\inf \left\{t \geq 0: \sigma_{t}^{\eta} \notin C(\eta)\right\} \\
\tau_{1}^{\eta} & =\inf \left\{t \geq \pi_{1}^{\eta}: \sigma_{t}^{\eta} \in M\right\} \\
\pi_{n}^{\eta} & =\inf \left\{t \geq \tau_{n-1}^{\eta}: \sigma_{t}^{\eta} \notin C\left(\sigma_{\tau_{n-1}^{\eta}}^{\eta}\right)\right\} \\
\tau_{n}^{\eta} & =\inf \left\{t \geq \pi_{n}^{\eta}: \sigma_{t}^{\eta} \in M\right\}
\end{aligned}
$$

$\operatorname{com} \pi_{0}^{\eta}=\tau_{0}^{\eta}=0$ 
Lema 4 Se $\eta$ é uma configuração em $M e$

$$
F(\eta)=\{\xi \notin C(\eta):<\xi, \rho>\text {, para algum } \rho \in \bar{C}(\eta)\}
$$

então para todo $k<\infty$ fixado

$$
\lim _{\beta \rightarrow \infty} P\left(\sigma_{\pi_{n}^{\eta}}^{\eta} \in F\left(\sigma_{\tau_{n-1}^{\eta}}^{\eta}\right) \text { para todo } n \leq k\right)=1
$$

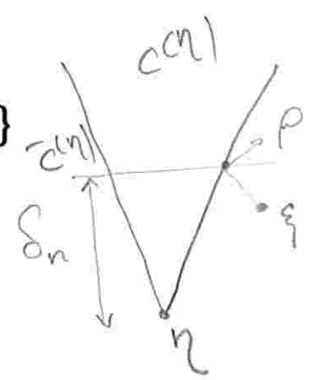

Dem : Como $k$ é finito, pela propriedade forte de Markov basta verificar o lema para $k=1$. Como $|\Sigma|<\infty$ existe um $a>0$ tal que, para duas configurações $\xi_{1}$ e $\xi_{2}$ quaisquer, vale

$$
H\left(\xi_{1}\right) \neq H\left(\xi_{2}\right) \Longrightarrow\left|H\left(\xi_{1}\right)-H\left(\xi_{2}\right)\right|>a
$$

Então

$$
\begin{aligned}
& P\left(\sigma_{\pi_{1}^{\eta}}^{\eta} \notin F(\eta)\right) \leq P\left(\pi_{1}^{\eta}>e^{\beta\left(\delta_{\eta}+\varepsilon\right)}\right)+ \\
& P\left(\tilde{\sigma}_{t}^{\eta} \in \partial C(\eta) \backslash \bar{C}(\eta) \text { para algum } t \leq e^{\beta\left(\delta_{\eta}+\epsilon\right)}\right)
\end{aligned}
$$

onde $\tilde{\sigma}_{t}^{\eta}$ é o processo restrito a $C(\eta)$ acoplado com $\sigma_{t}^{\eta}$ através do acoplamento A. O segundo termo de (1.8) vem do fato de se $\sigma_{\pi_{1}^{\eta}}^{\eta} \notin F(\eta)$ então a saida se deu por uma configuração de $\partial C(\eta) \backslash \bar{C}(\eta)$ e até $\pi_{1}^{\eta}$ ( exclusive ) os processos são idênticos. Este termo vai a zero por (1.7) e o Lema 2.

Quanto ao primeiro termo de (1.8) temos

$$
P\left(\pi_{1}^{\eta}>e^{\beta\left(\delta_{\eta}+\epsilon\right)}\right) \leq P\left(\tau_{1}^{\eta}>e^{\beta\left(\delta_{\eta}+\epsilon\right)}\right)
$$

que vai a zero pelo Lema 3 .

$\square_{\text {Lema } 4}$

Os resultados anteriores sugerem estudar a evolução de $\left\{\sigma_{t}^{\eta}\right\}_{t \geq 0}$ a partir do processo $\left\{X_{t}^{\eta}\right\}_{t \geq 0}$ definido por

$$
X_{t}^{\eta}=\sum_{n=0}^{\infty} \sigma_{\tau_{n}^{\eta}}^{\eta} \mathbb{1}_{\left[\tau_{n}^{\eta}, \tau_{n+1}^{\eta}\right)}(t)
$$

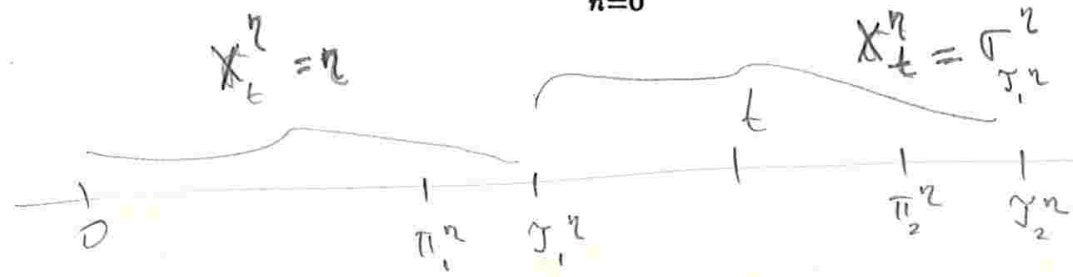


onde $\mathbb{I}_{I}(x)$ é a função indicador de $I \subset \mathbb{R}$.

De fato, uma primeira aplicação desse processo é o lema seguinte.

Lema 5 No caso fundamental, se $\eta \in M$ e $A=\left\{\eta \in M: \delta_{\eta} \leq(L-2) h\right\}$, então

$$
\lim _{\beta \rightarrow \infty} P\left(T_{X}^{\eta}\left(A^{c}\right)<e^{\beta[(L-2) h+\epsilon]}\right)=1
$$

onde $T_{X}^{\eta}\left(A^{c}\right)=\inf \left\{t \geq 0: X_{t}^{\eta} \notin A\right\}$.

Dem : Pelos Lemas 1 e 3 temos que, em A, cada transição de $X_{t}^{\eta}$ demora menos que $e^{\beta[(L-2) h+\delta]}$, para $\delta>0$ com probabilidade que vai a 1 quando $\beta$ cresce. Para mostrar o Lema 1.5 basta, então, provar que $X_{t}^{\eta}$ deixa $A$ num número de passos limitado superiormente com probabilidade que vai a 1 quando $\beta$ cresce e é tal que essa limitação não depende de $\beta$.

Se $\eta \notin A$ o lema é verdadeiro. Suponhamos, então, $\eta \in A$.

Seja $\rho \in \bar{C}(\eta)$ de forma que existe uma sequência $\xi_{0}=\rho, \xi_{1}, \ldots, \xi_{k}=\eta$, para algum k com $<\xi_{i}, \xi_{i+1}>$ e $H\left(\xi_{i+1}\right)<H\left(\xi_{i}\right)$. Como $0 \leq H\left(\xi_{i}\right)-H\left(\xi_{i+1}\right) \leq \delta_{\eta} \leq(L-2) h<$ $2-h$ para todo $i=0,1, \ldots, k-1$ a conclusão é que a variação de energia em cada transição foi de $h$, que corresponde a uma troca de spin +1 com d vizinhos com spin +1 .

Em $\rho$ existe um spin virando com taxa 1 cuja troca leva para fora de $C(\eta)$. Vamos mostrar que esse spin é +1 com d-1 vizinhos +1 .

Suponha, por absurdo, que esse spin, digamos no sítio $\mathrm{x}$, é -1 e seja $\bar{\rho}$ a configuração obtida de $\rho$ pela sua troca, ou seja

$$
\bar{\rho}= \begin{cases}\rho(y) & \text { se } y \neq x \\ -\rho(y) & \text { caso contrário }\end{cases}
$$

Sejam $x_{1}, x_{2}, \ldots, x_{k}$ as posições dos spins que trocam em cada transição da sequência $\xi_{0}, \xi_{1}, \ldots, \xi_{k}$. Se $x \neq y$ então $\bar{\rho}\left(x_{1}\right)=\rho\left(x_{1}\right)=-1$ e ambos viram com taxa 1 
( sua troca diminue a energia de $h$ ). Defina $\bar{\rho}_{1}$ como a configuração obtida de $\bar{\rho}$ pela troca do spin no ponto $x_{1}$. Iteramos esse argumento enquanto $x \neq x_{i}$ definindo $\bar{\rho}_{2}, \bar{\rho}_{3}, \ldots$ etc... até que $x=x_{j}$ para algum $j$. Mas isso necessariamente acontece para $j \leq k$ pois $\rho \leq \xi_{1} \leq \ldots \leq \xi_{k}=\eta$ de forma que o spin -1 em $x$ continuaria virando com taxa 1 em $\eta$ se $x \neq x_{i}$ para todo $i \in\{1, \ldots, k\}$ contrariando a hipótese $\eta \in M$.

No entanto quando $x=x_{j}$ para algum $j \in\{1, \ldots, k\}$ teremos $\xi_{j}=\bar{\rho}_{j-1}$ de forma que obtemos uma sequência de configurações que só diminue a energia ligando $\bar{\rho}$ e $\eta$ o que contraria a hipótese $\bar{\rho} \notin C(\eta)$.

Então a saida de $C(\eta)$ foi através da troca de um spin +1 . Em $\eta$ todo spin +1 tem pelo menos d vizinhos +1 . Como $\rho \in \bar{C}(\eta)$ foi atingido por uma sequência de trocas de spin +1 o primeiro spin +1 a virar com taxa 1 tem $d-1$ vizinhos +1 e se novamente chamamos $\bar{\rho}$ a configuração fora de $C(\eta)$ atingida pela sua troca, temos

$$
H(\rho)-H(\bar{\rho})=2-h
$$

Se $\bar{\rho} \notin M$ o processo $\sigma_{t}^{\bar{\rho}}$ vai atingir $M$ por transições que só diminuem a energia com probabilidade tendendo a 1 quando $\beta$ cresce ( Lema 1.1 ). Então

$$
\lim _{\beta \rightarrow \infty} P\left(H(\rho)-H\left(\sigma_{\tau_{1}^{\eta}}^{\eta}\right) \geq 2-h\right)=1
$$

Mas se $\sigma_{\tau_{1}^{\eta}}^{\eta} \in A$ e $H(\rho)-H\left(\sigma_{\tau_{1}^{\eta}}^{\eta}\right) \geq 2-h$ então

$$
\bar{C}\left(\sigma_{\tau_{1}^{\eta}}^{\eta}\right) \cap C(\eta)=\emptyset
$$

e o Lema 4 garante que $X_{t}^{\eta}$ não volta a $\eta$ em um passo com probabilidade tendendo a 1 quando $\beta$ cresce. Repetindo o argumento anterior para $\eta_{1}=\sigma_{\tau_{1}^{\eta}}^{\eta}$ obtemos uma sequência $\eta_{1}, \eta_{2}, \ldots$ de configurações em $M$. Como $|A|<\infty$ e por (1.12)

$$
\lim _{\beta \rightarrow \infty} P\left(H\left(\eta_{i}\right)-H\left(\eta_{i+1}\right) \geq 2-h-h(L-2)>0\right)=1
$$

concluimos que com probabilidade tendendo a 1 quando $\beta$ cresce a saida de $A$ ocorre em um número de passos não superior a $|A|$. 
Considere agora os conjuntos definidos por

$$
\begin{aligned}
\mathcal{M}_{1} & =\left\{\eta \in M: \delta_{\eta}>2-h\right\} \\
\mathcal{M}_{2} & =\left\{\eta \in M: \delta_{\eta}=2-h \text { e } \bar{C}(\eta) \cap\left(\cup_{\xi \in A^{c}} C(\xi)\right)=\emptyset\right\} \\
\mathcal{M} & =\mathcal{M}_{1} \cup \mathcal{M}_{2}
\end{aligned}
$$

Um exemplo de configuração em $\mathcal{M}_{1}$, em $\mathrm{d}=3$, é aquela na qual todos os spins são -1 exceto aqueles no interior de um paralelepípedo de lados $\mathrm{P}, \mathrm{Q}$ e $\mathrm{R}$ com todos os lados maiores que $2 / h$. Já as configurações de $\mathcal{M}_{2}$ podem ser bastante mais complicadas. Um exemplo simples de uma configuração desse conjunto seria o "halter" formado por dois paralepípedos de spins +1 com todos os lados maiores que $2 / h$ unidos por um "tubo" de spins +1 de secção retangular cujos lados são maiores que 2 e menores que $2 / h$.

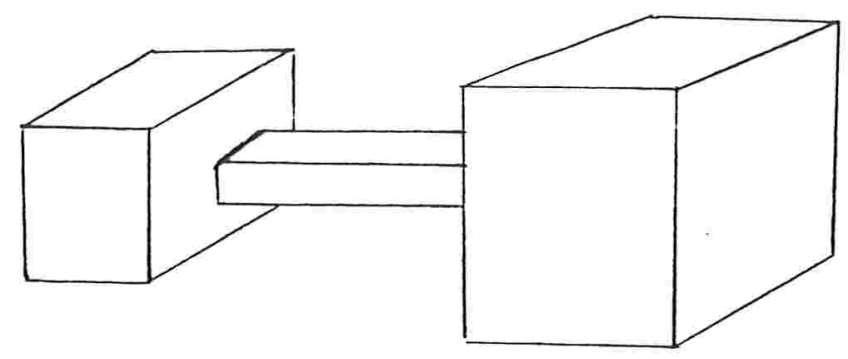

O que vamos mostrar em seguida é que, partindo de uma configuração qualquer, o processo atinge uma configuração de $\mathcal{M}$ num tempo inferior a $e^{\beta(2-h+\epsilon)}$ com probabilidade que vai a 1 quando $\beta$ cresce. Então, num certo sentido, essas configurações atraem as demais e cada configuração $\eta \in \mathcal{M}$ vai definir uma "bacia de atração" em $\Sigma$, que denotaremos por $B(\eta)$, da qual ela será o mínimo de energia. Dentro dessa bacia de atração existirão, no entanto, vários mínimos locais de energia correspondendo a configurações de $M$. Os dois tipos de configurações de $\mathcal{M}$ diferem nas características do cone de energia que elas definem. A partir de uma configuração de $\mathcal{M}_{1}$ não atingimos nenhuma configuração de $M$ num tempo inferior a $e^{\beta(2-h+\epsilon)}, \epsilon>0$ suficientemente pequeno, com probabilidade que vai a 1 quando $\beta$ cresce. Isto já não é verdade se a configuração inicial for de $\mathcal{M}_{2}$. Por exemplo, se a configuração inicial for aquela descrita anteriormente, poderíamos atingir nesse tempo a configuração com uma linha adicional de spins $+1 \mathrm{em}$ 
um dos cantos da junção dos paralelepípedos e o tubo.

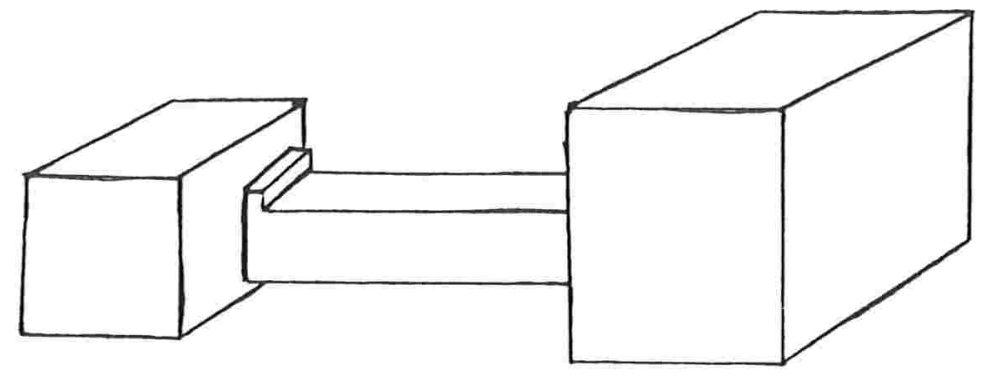

Essa configuração, digamos $\xi$, está em $M$ mas $\delta_{\xi} \leq(L-2) h$ uma vez que a linha adicional tem comprimento menor que $L$. A partir de $\xi$ o mais provável é que essa linha seja removida e retornemos à configuração inicial. Observe que o tempo gasto no retorno é muito pequeno, para $\beta$ grande, em relação àquele necessário para atingir $\xi$ de forma que a distribuição de probabilidades finito dimensionais definida pelo processo terá sua massa concentrada em $\xi$ para tempos menores que $e^{\beta(2-h+\epsilon)}, \epsilon>0$ suficientemente pequeno. Vamos mostrar agora que todas as configurações que podem ser alcançadas a partir de configurações de $\mathcal{M}_{2}$ com um custo energético de $2-h$ retornam rapidamente, na escala de tempo na qual ela é atingida, à configuração inicial. Esse resultado será crucial na demonstração do Lema 7 no qual se determina o tempo de relaxação na bacia de atração de uma configuração qualquer de $\mathcal{M}$.

Lema 6 No caso fundamental sejam $\eta \in M \operatorname{com} \delta_{\eta}=2-h$ e $\xi \in M$ tal que $H(\xi) \geq H(\eta)$ e $C(\xi) \cap \bar{C}(\eta) \neq \emptyset$. Então $\overline{C(\xi)} \cap C(\zeta)=\emptyset$ se $\zeta \neq \eta$.

Dem : Primeiro observe que se $\rho \in \bar{C}(\eta)$ então $\rho$ foi obtido de $\eta$ pela troca de um spin -1 com d-1 vizinhos +1 uma vez que, dada a hipótese de $2 / h \notin \mathbb{I}$, não existe outra transição ( ou sequência de transições) com aumento de energia de $2-h$. Seja $x_{0}$ a posição desse spin. Em $\rho \in \bar{C}(\eta)$ existe pelo menos um spin virando com taxa 1 cuja troca leva para fora de $C(\eta)$. Esse spin não pode ser +1 pois como $\rho \geq \eta$ todo spin +1 em $\rho$ que também é +1 em $\eta$ ( ou seja, todo, exceto aquele no sítio $x_{0}$ ) vira com taxa que vai a zero quando $\beta$ cresce e, se o spin em $x_{0}$ vira, voltamos a $\eta$. 
Como em $\eta$ todo spin -1 tem no máximo $d-1$ vizinhos +1 segue que um spin -1 em $\rho$ virando com taxa 1 levando para fora de $C(\eta)$ deve ter $\mathrm{d}$ vizinhos +1 e sua troca leva a uma configuração $\rho_{1}$ com

$$
H(\rho)-H\left(\rho_{1}\right)=h
$$

Note que se $x_{1}$ é a posição do spin que virou na transição para $\rho_{1}$ então $x_{1}$ é vizinho de $x_{0}$ e o spin +1 em $x_{0}$ passa a virar com taxa que vai a zero quando $\beta$ cresce.

Repetindo o argumento anterior podemos gerar uma sequência de configurações $\rho_{0}=\rho, \rho_{1}, \ldots, \rho_{k}$, para algum $k, \operatorname{com}<\rho_{i}, \rho_{i+1}>, \rho_{i} \leq \rho_{i+1}, H\left(\rho_{i}\right)>H\left(\rho_{i+1}\right)$ para $i=0,1, \ldots, k-1$ e $\rho_{k} \in M$.

Nas hipóteses do lema estamos interessados no caso $\rho_{k}=\xi \operatorname{com} H\left(\rho_{k}\right) \geq H(\eta)$ ( observe que, por construção, $C\left(\rho_{k}\right) \cap \bar{C}(\eta) \neq \emptyset$ ). No entanto basta considerar o caso $H\left(\rho_{k}\right)>H(\eta)$ uma vez que $H\left(\rho_{k}\right)-H(\eta)=k h-(2-h) \neq 0$ se $2 / h \notin I N$.

Esta condição implica $\delta_{\xi} \leq(L-2) h$ de forma que $H\left(\rho_{i}\right)-H\left(\rho_{i+1}\right)=h$ para todo $i=0,1, \ldots, k-1$. Sejam $x_{1}, x_{2}, \ldots, x_{k}$ as posições dos spins que viram ao longo da sequência. Vamos considerar agora a saida de $C(\xi)$.

Seja $\xi_{0}=\xi, \xi_{1}, \ldots, \xi_{l} \in \bar{C}(\xi)$ uma sequência de configurações com $\left\langle\xi_{i}, \xi_{i+1}\right\rangle$, $H\left(\xi_{i+1}\right)>H\left(\xi_{i}\right)$ e sejam $y_{1}, y_{2}, \ldots, y_{l}$ as posições dos spins que viram ao longo dessa sequência. Observe que, como $\rho \in \partial C(\xi)$, temos $l \leq k$.

A partir de $\eta$ defina $\eta_{1}$ como

$$
\eta_{1}(x)= \begin{cases}\eta(x) & \text { se } x \neq y_{1} \\ -1 & \text { caso contrário }\end{cases}
$$

de tal forma que, se $\eta\left(y_{1}\right)=-1$, então $\eta=\eta_{1}$, caso contrário temos $\left\langle\eta, \eta_{1}>\mathrm{e}\right.$ $0 \leq H\left(\eta_{1}\right)-H(\eta) \leq h$ uma vez que $\eta \leq \xi$. De forma análoga, podemos definir $\eta_{2}, \eta_{3}, \ldots, \eta_{l}$. Como $\xi_{l} \in \bar{C}(\xi)$ existe um spin +1 , digamos na posição $y_{l+1}$, virando com taxa 1 cuja troca leva para fora de $C(\xi)$ ( vide demonstração do Lema 5 ). Se esse spin também for +1 em $\eta_{l}$ ele também vira com taxa 1 nesta configuração ( pois $\eta_{l} \leq \xi_{l}$ ). Então definimos 
$\eta_{1+1}$ como

$$
\eta_{l+1}(x)= \begin{cases}\eta(x) & \text { se } x \neq y_{l+1} \\ -1 & \text { caso contrário }\end{cases}
$$

Agora, como $l \leq k$, se $\eta_{l+1} \neq \eta$ então existe uma saida de $C(\eta)$ com custo energético menor que $2-h$ violando a hipótese $\delta_{\eta}=2-h$. Então $\eta_{l+1}=\eta$ e $\left\{y_{1}, y_{2}, \ldots, y_{l+1}\right\}=$ $\left\{x_{0}, \ldots, x_{k}\right\}$, e o lema está demonstrado.

Provamos agora o resultado que caracteriza as configurações de $\mathcal{M}$ como "centro de bacias de atração", no sentido de que a partir de uma configuração qualquer atingimos um elemento de $\mathcal{M}$ num tempo da ordem de $e^{\beta(2-h+\epsilon)}, \epsilon>0$.

Lema 7 No caso fundamental se $\eta \in \Sigma e \epsilon>0$

$$
\lim _{\beta \rightarrow \infty} P\left(T^{\eta}(\mathcal{M})<e^{\beta(2-h+\epsilon)}\right)=1
$$

Dem : Pelo Lema 1 basta considerar $\eta \in M$ de forma que (1.19) segue se provarmos que para $\eta \in M$ e $\delta>0$

$$
\lim _{\beta \rightarrow \infty} P\left(T_{X}^{\eta}(\mathcal{M})<e^{\beta(2-h+\delta)}\right)=1
$$

Pelo Lema 5 basta considerar $\eta \in M \backslash(A \cup \mathcal{M})$, ou seja, $\eta$ tal que

$$
\delta_{\eta}=2-h \text { e } \bar{C}(\eta) \cap\left(\cup_{\zeta \in A^{c}} C(\zeta)\right) \neq \emptyset
$$

Tomemos então $\eta$ satisfazendo (1.21). Seja $\xi$ a configuração em $M$ atingida após a saida de $C(\eta)$ através de uma configuração de $\bar{C}(\eta)$. Se $H(\xi)>H(\eta)$ o Lema 6 garante que, com probabilidade que vai a 1 quando $\beta$ cresce, o processo retorna a $\eta$. Como toda configuração de $\bar{C}(\eta)$ tem probabilidade maior ou igual a $N^{-d}$ de ser atingida em 1 passo a partir de $\eta$ e vale (1.21) então um $\xi \in M \operatorname{com} H(\xi)<H(\eta)$ será atingido através de $\bar{C}(\eta)$ num tempo inferior a $e^{\beta(2-h+\epsilon)}, \epsilon>0$, com probabilidade que vai a 1 quando $\beta$ cresce. Podemos ter três situações: 
1) Se $\delta_{\xi}>2-h$ então $\xi \in \mathcal{M}$ e o lema é satisfeito nesse caso.

2) Se $\delta_{\xi}<2-h$ e portanto $\delta_{\xi} \leq(L-2) h$ então o Lema 5 garante que uma configuração de $M \backslash(A \cup\{\eta\})$ com energia inferior àquela de $\eta$ será atingida, com probabilidade que vai a 1 quando $\beta$ cresce, num tempo inferior a $e^{\beta((L-2) h+c)}$ que é muito menor que $e^{\beta(2-h+c)}$ para $\beta$ grande. Essa nova configuração pode satisfazer 1) ou satisfazer a condição 3 ) apresentada abaixo.

3) Se $\delta_{\xi}=2-h$ temos duas possibilidades.

3a) Se vale (1.21) então repetimos o procedimento anterior com $\xi$ tomando o lugar de $\eta$. Note que, como $H(\xi)<H(\eta)$ e $|\Sigma|<\infty$, essa iteração não pode continuar indefinidamente e de fato termina num tempo inferior a $e^{\beta(2-h+\epsilon)}, \epsilon>0$ com probabilidade tendendo a 1 quando $\beta$ cresce. Essa sequênca terminar significa atingir uma configuração satisfazendo 1) ou uma satisfazendo 3 b) abaixo.

3b) Caso $\delta_{\eta}=2-h$ mas (1.21) não vale então $\xi \in \mathcal{M}$ e o lema é válido também nesse caso.

Os lemas anteriores permitem caracterizar $\mathcal{M}$ como mínimos de energia num sentido mais forte que aquele usado na definição de $M$. Para cada elemento $\eta \in \mathcal{M}$ podemos definir sua bacia de atração como sendo o conjunto de todas as configurações que têm uma probabilidade que não vai a zero quando $\beta$ cresce de entrar em $\mathcal{M}$ pela primeira vez através de $\eta$. Mais precisamente:

Definição : Bacia de atração de $\eta \in \mathcal{M}$ :

$$
B(\eta)=\left\{\rho \in \Sigma: \text { existe } \epsilon>0 \text { tal que } P\left(\sigma_{T^{\rho}(\mathcal{M})}^{\rho}=\eta\right)>\epsilon \text { para todo } \beta>0\right\}
$$

E, analogamente às definições para os cones de uma configuração, temos:

Definição : Fronteira da bacia de $\eta \in \mathcal{M}$ :

$$
\partial B(\eta)=\{\xi \in B(\eta) \cap B(\rho) \text { para } \rho \in \mathcal{M} \backslash\{\eta\}\}
$$


Definição : Altura da fronteira de $\eta \in \mathcal{M}$ :

$$
\Delta_{\eta}=\min \{H(\xi)-H(\eta) \text { para } \xi \in \partial B(\eta)\}
$$

A estrutura de mínimos e barreiras de energia desenvovida neste capítulo se baseou fortemente das possíveis variações de energia e que à cada variação correspondesse um único tipo de transição. Nas aplicações desses resultados para dimensão três apresentadas no Capítulo 2 usaremos a estrutura de mínimos e barreiras de energia não de $\left\{\sigma_{t}^{\eta}\right\}_{t \geq 0}$ mas de um outro processo, $\left\{\widehat{\sigma}_{t}^{\eta}\right\}_{t \geq 0}$ no qual certos spins estão congelados, i.e. viram com taxa 0 . Como isso equivale a tomar uma variação de energia infinita para certas transições, não existe qualquer problema em definir para esse processo a bacia de atração de uma configuração a qual será denotada por $\widehat{B(\eta)}$ para lembrar que se refere ao processo modificado. Da mesma forma escreveremos $\partial \widehat{B(\eta)}$ para a fronteira da bacia desse processo. 


\section{Capítulo 2}

\section{Caso subcrítico tridimensional}

Provamos aqui o Teorema 4.

Neste capítulo $\eta$ sempre será usado para denotar a configuração em $\mathcal{R}$ na qual todos os spins são -1 exceto aqueles no interior do paralelepípedo de lados $P, Q$ e $R$ com $P \leq Q \leq R$. Se $P<L$ então $\eta \notin \mathcal{M}$, uma vez que $\delta_{\eta} \leq(L-2) h$ e portanto $\eta \in A$. Nesse caso o Lema 5 garante que uma configuração fora de $A$ é atingida antes de um tempo $e^{\beta[(L-2) h+\epsilon]}, \epsilon>0$, com probabilidade que vai a 1 quando $\beta$ cresce. É imediato verificar que essa configuração em $M \backslash A$ é $-\underline{1}$. Como $E(h) \geq(L-2) h$ segue a limitação no tempo.

Consideramos então, a partir de agora, $\eta \operatorname{com} L \leq P \leq Q \leq R$.

Seja $\left\{\hat{\sigma}_{t}^{\eta}\right\}_{t \geq 0}$ o processo no qual todos os spins +1 dentro do paralelepípedo definido por $\eta$ estão congelados exceto aqueles de uma das fatias externas de lados $P$ e $Q$. Para sermos mais específicos suponhamos, sem perda de generalidade, que o paralelepípedo definido por $\eta$ consiste nos sítios de $R:=\{1, \ldots, P\} \times\{1, \ldots, Q\} \times\{1, \ldots, R\}$. Então $\left\{\widehat{\sigma}_{t}^{\eta}\right\}_{t \geq 0}$ é o processo com configuração inicial $\eta$ e taxas

$$
\hat{c}(x, \xi)= \begin{cases}0 & \text { se } x \in\{1, \ldots, P\} \times\{1, \ldots, Q\} \times\{1, \ldots, R-1\} \\ c(x, \xi) & \text { caso contrário }\end{cases}
$$


Estabelecendo entre $\left\{\sigma_{t}^{\eta}\right\}_{t \geq 0}$ e $\left\{\widehat{\sigma}_{t}^{\eta}\right\}_{t \geq 0}$ o acoplamento básico ou de Vaserstein [Lig], temos

$$
P\left(\sigma_{t}^{\eta} \leq \hat{\sigma}_{t}^{\eta}, \text { para todo } t \geq 0\right)=1
$$

O lema seguinte mostra que esse novo processo atinge uma configuração de $\mathcal{M} \backslash\{\eta\}$ num tempo menor que $e^{\beta(E(h)+\epsilon)}$. Usamos em sua demonstração os resultados obtidos no capítulo I para o processo $\left\{\sigma_{t}^{\eta}\right\}_{t \geq 0}$ mas que também são válidos para $\left\{\widehat{\sigma}_{t}^{\eta}\right\}_{t \geq 0}$.

Lema 8 Se $\eta \in \mathcal{R}$ é tal que $L \leq P \leq Q \leq R$ e $Q<Q_{c}$ no caso fundamental então para todo $\epsilon>0$

$$
\lim _{\beta \rightarrow \infty} P\left(\hat{T}^{\eta}(\mathcal{M} \backslash\{\eta\})<e^{\beta(E(h)+4 \epsilon)}\right)=1
$$

onde, para $A \subset \Sigma, \widehat{T}^{\eta}(A)=\inf \left\{t \geq 0: \widehat{\sigma}_{t}^{\eta} \in A\right\}$ e $Q_{c}=2 P /(P h-2)$ é o valor crítico heurístico obtido na introdução.

O primeiro passo para provar (2.3) consiste em determinar uma boa limitação superior para a probabilidade desse processo deixar a bacia de atração de $\eta$ durante um intervalo de tempo igual ao tempo de relaxação do sistema nessa bacia. Essa limitação é obtida no próximo lema, que demonstramos antes de provar o Lema 8.

Lema 9 Se $\eta \in \mathcal{R}$ é tal que $L \leq P \leq Q \leq R$ e $Q<Q_{c}$ no caso fundamental então para todo $\xi \in \widehat{B}(\eta)$ e $\epsilon>0$ existe $\beta_{0}<\infty$ tal que

$$
P\left(\widehat{T}^{\xi}\left(\widehat{B}^{c}(\eta)\right) \leq e^{\beta(2-h+3 \epsilon)}\right) \geq \frac{1}{3} e^{-\beta(E(h)-2+h)}
$$

para todo $\beta>\beta_{0}$ onde $\widehat{B}(\eta)$ é a bacia de atração de $\eta$ definida pelo processo $\left\{\widehat{\sigma}_{t}^{\eta}\right\}_{t \geq 0}$ como no capítulo $I$.

Dem : Seja $\zeta$ uma das possíveis configurações obtidas de $\eta$ removendo os spins +1 da fatia $\{1, \ldots, P\} \times\{1, \ldots, Q\} \times\{R\}$ e sobre esta acrescentando uma proto-gota crítica bidimensional. Então $\zeta \in \partial \hat{B}(\eta)$. 
Isto porque se o (único) spin +1 virando com taxa 1 o fizer chegamos a uma configuração em $M$ e fora de $\hat{B}(\eta)$; por outro lado se um dos spins -1 virando com taxa 1 mudar de sinal entramos no cone de uma configuração, correspondendo a um paralelepípedo de lados $P, Q$ e $R-1$ com um quadrado $L \times L$ em uma das faces, que retorna a $\eta$.

Seja $\underline{\zeta}$ a configuração obtida de $\zeta$ pela troca daquele spin +1 que vira com taxa 1. Observe que $H(\zeta)-H(\underline{\zeta})=2-h$.

Considere o conjunto $\mathcal{F}=\widehat{B}(\eta) \cup\{\underline{\zeta}\}$ e sejam $\left\{\tilde{\sigma}_{t}^{\eta}\right\}_{t \geq 0}$ o processo obtido de $\left\{\hat{\sigma}_{t}^{\eta}\right\}_{t \geq 0}$ pela restrição a $\mathcal{F}$ e $\tilde{\mu}$ sua medida invariante (ou seja, aquela dada por (1.1) com $\mathcal{S}=\mathcal{F})$.

Se $\xi \in \partial \widehat{B}(\eta)$ então (2.4) é verdadeiro pois o processo sai dessa configuração com taxa maior que 1 e a probabilidade de ocorrer uma particular transição levando para fora de $\widehat{B}(\eta)$ é maior ou igual a $N^{-3}$. Então, nesse caso $(\xi \in \partial \widehat{B}(\eta))$, temos

$$
P\left(\widehat{T}^{\xi}\left(\hat{B}^{c}(\eta) \leq e^{\beta(2-h+3 \epsilon)}\right) \geq \frac{1-e^{-e^{\beta(2-h+3 \epsilon)}}}{N^{3}}\right.
$$

implicando (2.4) para $\beta$ suficientemente grande.

Suponhamos então $\xi \in \widehat{B}(\eta) / \partial \widehat{B}(\eta)$. Nesta situação o Lema 5 fornece que, para todo $\delta>0$

$$
\lim _{\beta \rightarrow \infty} P\left(\widehat{T}^{\xi}\left(\widehat{B}^{c}(\eta)\right)>\widehat{T}^{\xi}(\eta), T^{\xi}(\eta)<e^{\beta(2-h+c)}\right)=1
$$

Então

$$
\begin{aligned}
& P\left(\widehat{T}^{\xi}\left(\hat{B}^{c}(\eta)\right) \leq e^{\beta(2-h+3 \epsilon)}\right) \geq \\
& P\left(\widehat{T}^{\xi}(\eta)<\widehat{T}^{\xi}\left(\widehat{B}^{c}(\eta)\right) ; \widehat{T}^{\xi}(\eta)<e^{\beta(2-h+\epsilon)} ; \widehat{T}^{\xi}\left(\hat{B}^{c}(\eta)\right) \leq e^{\beta(2-h+2 \epsilon)}\right)= \\
& P\left(\widehat{T}^{\xi}(\eta)<\widehat{T}^{\xi}\left(\hat{B}^{c}(\eta)\right) ; \widehat{T}^{\xi}(\eta)<e^{\beta(2-h-\epsilon)}\right) P\left(\hat{T}^{\eta}\left(\widehat{B}^{c}(\eta)\right) \leq e^{\beta(2-h+2 \epsilon)}\right) \geq
\end{aligned}
$$

OBS Em fo 0 tempo do relaraces a $N e^{\beta(\beta-h)}$, is to é;

$$
?\left(\tilde{T}\left\{(\eta)<e^{\beta(z-h+f)}\right) \rightarrow 1 \quad \forall \delta>0 .\right.
$$




$$
\frac{1}{2} P\left(\widehat{T}^{\eta}\left(\widehat{B}^{c}(\eta)\right) \leq e^{\beta(2-h+2 \epsilon)}\right)
$$

para $\beta$ suficientemente grande. Na segunda passagem usamos a propriedade forte de Markov e na última usamos (2.5).

A conclusão é que basta mostrar (2.4) para $\xi=\eta$.

Agora usamos o acoplamento $A$ entre os processos $\left\{\widehat{\sigma}_{t}^{\eta}\right\}_{t \geq 0}$ e $\left\{\tilde{\sigma}_{t}^{\eta}\right\}_{t \geq 0}$. Se defin$\operatorname{imos} \tilde{T}^{\eta}=\inf \left\{t \geq 0: \tilde{\sigma}_{t}^{\eta} \notin \widehat{B}(\eta)\right\}$ e tomamos $V=e^{\beta(2-h+2 \epsilon)}$ temos:

$$
P\left(\widehat{T}^{\eta}\left(\widehat{B}^{c}(\eta)\right)>V\right) \leq P\left(\tilde{T}^{\eta}\left(\hat{B}^{c}(\eta)\right)>V\right)
$$

Tomamos agora o processo $\left\{\tilde{\sigma}_{t}^{\mu}\right\}_{t \geq 0}$ restrito a $\mathcal{F}$ e com configuração inicial escolhida conforme a medida $\tilde{\mu}$ e o acoplamos com $\left\{\tilde{\sigma}_{t}^{\eta}\right\}_{t \geq 0}$ através do acoplamento $B$ de forma que

$$
P\left(\tilde{T}^{\eta}\left(\hat{B}^{c}(\eta)\right)>V\right) \leq P\left(\tilde{\sigma}_{t}^{\eta} \neq \tilde{\sigma}_{t}^{\tilde{\mu}} \text { para } 0 \leq t \leq V\right)+P\left(\tilde{\sigma}_{V}^{\tilde{\mu}} \in \hat{B}(\eta)\right)
$$

Se $W=e^{\beta(2-h+\epsilon)}$ temos

$$
P\left(\tilde{\sigma}_{t}^{\eta} \neq \tilde{\sigma}_{t}^{\tilde{\mu}}, 0 \leq t \leq V\right) \leq\left(\sup _{\xi, \rho \in \mathcal{F}} P\left(\tilde{\sigma}_{t}^{\xi} \neq \tilde{\sigma}_{t}^{\rho}, 0 \leq t \leq W\right)\right)^{\left[e^{\beta e}\right]}
$$

onde $\widetilde{\sigma}_{t}^{\xi}$ e $\tilde{\sigma}_{t}^{\rho}$ são processos independentes.

Mas

$$
\sup _{\rho, \xi \in \mathcal{F}} P\left(\tilde{\sigma}_{t}^{\xi} \neq \tilde{\sigma}_{t}^{\rho}, 0 \leq t \leq W\right) \leq\left[\sup _{\rho \in \mathcal{F}} P\left(\tilde{\sigma}_{W}^{\rho} \neq \eta\right]^{2}\right)
$$

O termo do lado direito em (2.10) vai a zero quando $\beta$ cresce pois, pelo Lema 6 , o processo atinge $\eta$ num tempo inferior a $\frac{1}{2} e^{\beta(2-h+\epsilon)}$; como $\delta_{\eta}>2-h$ o processo 
permanece no cone de $\eta$ durante um tempo superior a $e^{\beta(2-h+\epsilon)}, \epsilon>0$ pequeno o que justifica a afirmação.

Usando esse fato em (2.9) temos que existe um $\beta_{1}>0$ tal que, para $\beta>\beta_{1}$

$$
\left[\sup _{\rho, \xi \in \mathcal{F}} P\left(\tilde{\sigma}_{t}^{\xi} \neq \tilde{\sigma}_{t}^{\rho}, 0 \leq t \leq W\right)\right]^{\left[e^{\beta \varepsilon}\right]} \leq[1 / 2]^{\left[e^{\beta \epsilon}\right]}
$$

Já quanto ao segundo termo de (2.8)

$$
P\left(\tilde{\sigma}_{V}^{\tilde{\mu}} \notin \hat{B}(\eta)\right)=\tilde{\mu}(\underline{\zeta})=e^{-\beta(E(h)-2+h)}\left[\sum_{\xi \in \mathcal{F}} e^{-\beta(H(\xi)-H(\eta))}\right]^{-1} \geq \frac{1}{2} e^{-\beta(E(h)-2+h)}
$$

para $\beta>\beta_{2} \operatorname{com} \beta_{2}<\infty$ suficientemente grande.

Usando (2.11) e (2.12) em (2.8), temos

$$
P\left(\tilde{T}^{\eta}\left(\hat{B}^{c}(\eta)\right) \leq V\right) \geq \frac{1}{2} e^{-\beta(E(h)-2+h)}-(1 / 2)^{\left\lfloor e^{\beta \epsilon}\right\rfloor} \geq \frac{1}{3} e^{-\beta(E(h)-2+h)}
$$

para $\beta>\beta_{0}$, com $\beta_{0}<\infty$ suficientemente grande.

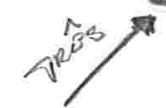

De posse desse resultado podemos passar à demonstração do Lema 8 .

$$
\begin{aligned}
& P\left(\widehat{T}^{\eta}(\mathcal{M} \backslash\{\eta\})>e^{\beta(E(h)+4 \epsilon)}\right) \leq \\
& \left.P\left(\hat{T}^{\eta}(\mathcal{M} \backslash\{\eta\})\right)<e^{\beta(E(h)+4 \epsilon)} ; \widehat{T}^{\eta}\left(\widehat{B}^{c}(\eta)\right)<e^{\beta(E(h)+4 \epsilon)}-e^{\beta(2-h+3 \epsilon)}\right)+ \\
& P\left(\hat{T}^{\eta}\left(\widehat{B}^{c}(\eta)\right)>e^{\beta(E(h)+4 \epsilon)}-e^{\beta(2-h+3 \epsilon)}\right)
\end{aligned}
$$

O primeiro termo de (2.14) vai a zero pelo Lema 6. Para o segundo termo, considere os tempos: 


$$
t_{i}=i e^{\beta(2-h+3 \epsilon)} \quad, \quad i=1,2, \ldots
$$

\section{Então}

$$
\begin{aligned}
& P\left(\hat{T}^{\eta}\left(\hat{B}^{c}(\eta)\right)>e^{\beta(E(h)+4 \epsilon)}-e^{\beta(2-h+3 \epsilon)}\right) \\
& \leq\left[\sup _{\xi \in B(\eta)} P\left(\hat{T}^{\eta}(\hat{B}(\eta))>e^{\beta(2-h+3 \epsilon)}\right)\right]^{e^{\alpha E(h)-2+h+\epsilon)}-2}
\end{aligned}
$$

que vai a zero quando $\beta$ cresce pelo Lema 2.2 .

Já temos uma limitação superior para o tempo até que o processo $\left\{\widehat{\sigma}_{t}^{\eta}\right\}_{t \geq 0}$ alcance uma outra configuração de $\mathcal{M}$ a partir de $\eta$. Vamos verificar agora que essa configuração atingida não é maior que $\eta$.

Defina $E=\{\xi \in \mathcal{M} \backslash\{\eta\}:|\xi / \eta|>0\}$ onde, se $\rho \in \Sigma,|\rho|=$ número de spins +1 em $\rho$. Então

$$
=\sum_{x \in \Lambda_{N}} \frac{1+p(x)}{2}
$$

Lema 10 No caso fundamental com $\eta$ tal que $L \leq P \leq Q \leq R$ e $Q<Q_{c}$ e $\epsilon>0$

$$
\lim _{\beta \rightarrow \infty} P\left(\widehat{T}^{\eta}(E)<e^{\beta(\Gamma(h)-\epsilon)}\right)=0
$$

Antes de demonstrar esse Lema precisamos de alguns resultados adicionais.

Sejam $\eta_{i}, i=1,2$ e 3 as seguintes configurações.

$$
\eta_{i}\left(x_{1}, x_{2}, x_{3}\right)= \begin{cases}+1 & \text { se } 1 \leq x_{i} \leq k_{i} \\ -1 & \text { caso contrário }\end{cases}
$$

onde $k_{1}=P, k_{2}=Q \mathrm{e} k_{3}=R$ e considere os eventos

$$
E_{i}=\left\{\xi \in E:\left|\xi / \eta_{i}\right|>0\right\} \quad i=1,2 \text { e } 3
$$

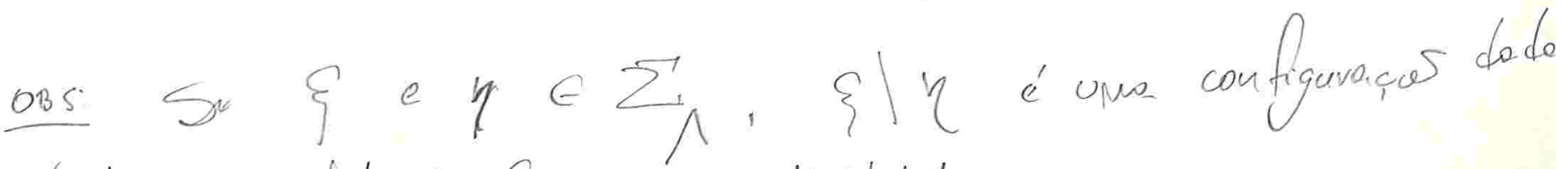

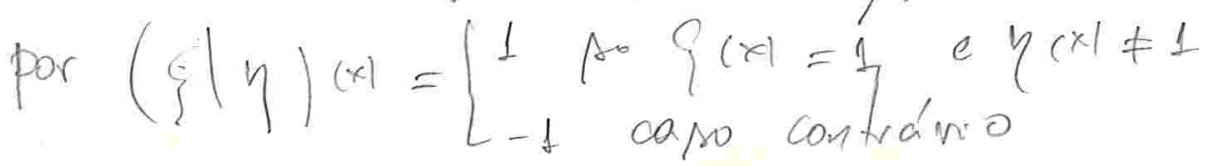




\section{Então}

$$
P\left(\widehat{T}^{\eta}(E)<e^{\beta(\Gamma(h)-\epsilon)}\right) \leq \sum_{i=1}^{3} P\left(\hat{T}^{\eta}\left(E_{i}\right)<e^{\beta(\Gamma(h)-\epsilon)}\right)
$$

Para $i=1,2$ ou 3 , fixado, considere o processo $\left\{\sigma_{t}^{\eta_{i}}\right\}_{t \geq 0}$, com configuração inicial $\eta_{i}$ e no qual todos os spins +1 em $\eta_{i}$ estão congelados (ou seja, viram com taxa 0 ), acoplado com $\left\{\hat{\sigma}_{t}^{\eta}\right\}_{t \geq 0}$ através do acoplamento básico de forma que

$$
P\left(\sigma_{t}^{\eta_{i}} \geq \hat{\sigma}_{t}^{\eta} \text { para todo } t\right)=1
$$

Vamos provar o Lema 10 verificando primeiramente que existe uma barreira de energia de altura $\Gamma(h)$ entre $\eta$ e $E_{i}$. Pelo Lema 2 segue que o processo $\left\{\sigma_{t}^{\eta_{i}}\right\}_{t \geq 0}$ não consegue atingir $E_{i}$ num tempo inferior a $e^{\beta(\Gamma(h)-\epsilon)}$ o que, por (2.18), implica (2.15).

Essa barreira que deve ser atravessada para chegar a $E_{i}$ é formada pelo seguinte conjunto:

$$
\mathcal{P}_{i}=\left\{\xi \geq \eta_{i}:\left|\xi \backslash \eta_{i}\right|=L(L-1)+1\right\}
$$

Para mostrar que é impossível passar de $\eta$ a $E_{i}$ sem atravessar $\mathcal{P}_{i}$ basta verificar que se $\xi$ é tal que $0<\left|\xi / \eta_{i}\right| \leq L(L-1)+1$ então $\xi \notin \mathcal{M}$. Para isto definimos

$$
\rho_{j}\left(x_{1}, x_{2}, x_{3}\right)= \begin{cases}+1 & \text { se } x_{i}=j \\ -1 & \text { caso contrário }\end{cases}
$$

e, para $\xi \in \Sigma, \xi_{j}=\xi \cap \rho_{i} .=$ parto do conf. $\{$ na Jépiou fatio na divers $i$.

Se $\xi$ é tal que $0<\left|\xi \backslash \eta_{i}\right| \leq L(L-1)+1$ então

$$
0<\sum_{j=k_{i}} N\left|\xi_{j}\right| \leq L(L-1)+1
$$

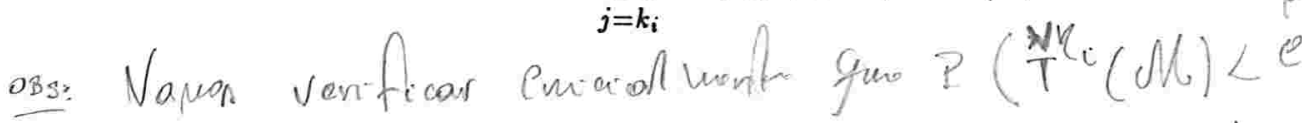

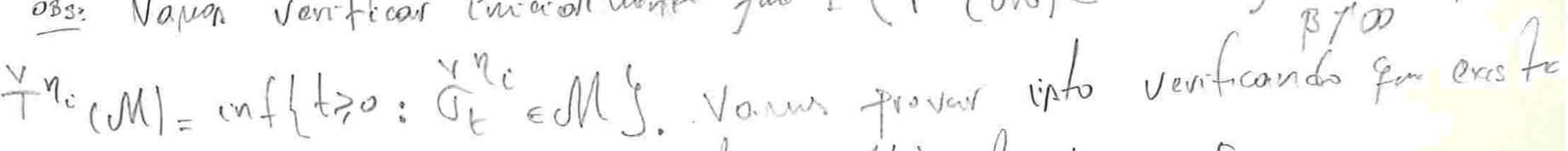
una "Barreivo de enorgen" de olltura p(h) entro $y$ e $E_{i}$.

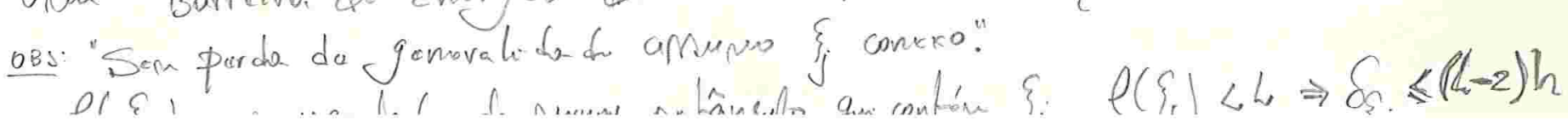



$\left.\xi_{i} \mid=0\right\}$.

De forma que, se $N>N(h)$ (caso fundamental), existe $m=\min \left\{j \in\left\{k_{i}, \ldots, N\right\}: \mid\right.$

Considere $\xi_{m-1}$. Essa configuração tem menos que $L^{2}$ spins +1 no plano definido por $x_{i}=m-1$ de forma que mesmo se $\xi_{m-2}=\rho_{m-2}$ (a camada anterior estivesse completa) teríamos $\delta_{\xi} \leq(L-2) h$ o que implica $\xi \notin \mathcal{M}$.

Considere agora o conjunto conexo de configurações $\mathcal{H}_{i}$ definido por

$$
\mathcal{H}_{i}=\left\{\xi \in \Sigma: \xi \geq \eta_{i} \text { e }\left|\xi \backslash \eta_{i}\right| \leq L(L-1)+1\right\}
$$

Então

Lema 11 No caso fundamental

$$
\text { para todo } \xi \in \mathcal{H}_{i} \operatorname{com} \xi \neq \eta^{i} \text { temos } H(\xi)>H\left(\eta_{i}\right)
$$

\section{Dem :}

Defina agora $H_{j}^{i}(\xi)$ como sendo contribuição da energia de $\xi$ levando-se em conta apenas as interações no plano $\left\{x \in \Lambda_{N}: x_{i}=j\right\}$, isto é:

$$
H_{j}^{i}(\xi)=-\frac{1}{2} \sum_{\left\langle x, y>: x, y \in X_{j}\right.} \xi(x) \xi(y)-\frac{h}{2} \sum_{x \in X_{i}} \xi(x)
$$

onde $X_{j}=\left\{x=\left(x_{1}, x_{2}, x_{3}\right) \in \Lambda_{N}: x_{i}=j\right\}$.

Então

$$
H(\xi)-H\left(\eta_{i}\right) \geq \sum_{j=k_{i}}^{N}\left[H_{j}^{i}(\xi)-H_{j}^{i}\left(\eta_{i}\right)\right]
$$

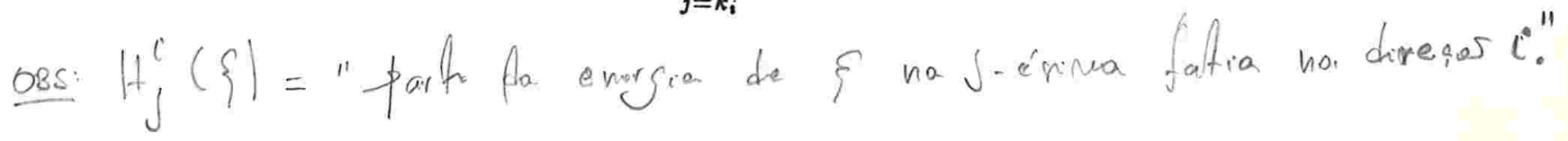


Verifiquemos essa desigualdade. Temos

$$
H(\xi)-H\left(\eta_{i}\right)=\sum_{j=1}^{N}\left[H_{j}^{i}(\xi)-H_{j}^{i}\left(\eta_{i}\right)\right]-\frac{1}{2} \sum_{x \in \Lambda_{N}}\left[\xi(x) \xi\left(x+e_{i}\right)-\eta_{i}(x) \eta_{i}\left(x+e_{i}\right)\right]
$$

onde no segundo termo no lado direito de (2.24) se $x \in \Lambda_{N}$ então $x+e_{i}$ é o seu vizinho mais próximo na direção $i$ na rede. Observe que esse termo é não negativo pois a configuração $\xi$ possui pelo menos tantos pares de vizinhos mais próximos na direção $i$ com sinais opostos quanto aqueles que possui $\eta_{i}$. Com isso e com o fato de, por hipótese $\xi \geq \eta_{i}$, de forma que $H_{j}^{i}(\xi)-H_{j}^{i}\left(\eta_{i}\right)=0 \quad \forall j \in\left\{1, \ldots, k_{i}-1\right\}$ obtemos $(2.23)$.

Para cada $j$ fixado o termo entre colchetes no lado direito de (2.23) representa a diferença de energia em relação ao estado fundamental de $\xi_{j}$ considerado como uma configuração bidimensional (no plano $X_{j}$ ). Vamos considerar cada um desses sistemas bidimensionais verificando que essa diferença de energia é não negativa de onde seguirá (2.21).

Para todo $j=k_{j}, k_{j+1}, \ldots, N$ temos $\left|\xi_{j}\right| \leq L(L-1)+1$. Podemos transformar a configuração $\xi_{i}$ numa outra configuração $\zeta_{i}$ com um único aglomerado de spins +1 transladando cada aglomerado de $\xi_{i}$, sem deformá-lo, até que todos se toquem. Essa nova configuração tem energia inferior àquela de $\xi_{j}$.

Como $N>N(h)$ o aglomerado único de $\zeta_{j}$ não pode circundar o toro $\Lambda_{N}$. Sejam $M_{j}$ e $N_{j}$ os lados do menor retângulo que engloba esse aglomerado. Seja $S_{j}=\left|\xi_{j}\right|$ $=$ número de spins +1 de $\xi$ no plano $X_{j}$. Então

$$
H_{j}^{i}(\xi)-H_{j}^{i}\left(\eta_{i}\right) \geq-h S_{j}+2\left(M_{j}+N_{j}\right)
$$

Como $M_{j} N_{j} \geq S_{j}$ com ambos positivos temos que $M_{j}+N_{j} \geq 2 \sqrt{S_{j}}$. Usando este fato em (II.23) e (II.21), obtemos 


$$
\begin{aligned}
& H(\xi)-H\left(\eta_{i}\right) \geq \sum_{j=k_{i}}^{N}\left[-h S_{j}+4 \sqrt{S_{j}}\right]= \\
& -h\left|\xi / \eta_{i}\right|+\sum_{j=K_{i}}^{N} 4 \sqrt{S_{j}} \geq-h\left|\xi / \eta_{i}\right|+4 \sqrt{\left|/ \eta_{i}\right|}
\end{aligned}
$$

que é não negativo uma vez que $0<\left|\xi / \eta_{i}\right|<L(L-1)+1$ implica $0<\sqrt{\left|\xi / \eta_{i}\right|}<4 / h$. $\square_{\text {Lema } 11}$

Vamos determinar agora a altura da barreira energética que o processo precisa vencer para deixar $\mathcal{H}_{i}$. Mais precisamente, obteremos uma limitação inferior para as energias das configurações em $\mathcal{P}_{i} \subset \mathcal{H}_{i}$.

Lema $12 S \epsilon \xi \in \mathcal{P}_{i}$ no caso fundamental então

$$
H(\xi)-H\left(\eta_{i}\right) \geq \Gamma(h)
$$

Dem : Novamente utilizamos a decomposição dada por (2.23). De (2.26) concluímos que uma limitação inferior para a diferença de energias em (2.27) é obtida considerandose um sistema bidimensional com os $L(L-1)+1$ spins +1 externos a $\eta_{i}$ num único aglomerado plano. Basta mostrar, então, que se $\rho$ é uma configuração bidimensional com $|\rho|=L(L-1)+1$ então

$$
H(\rho)-H(-1) \geq \Gamma(h)
$$

Para verificar essa relação procederemos como na demonstração do Lema 11 transformando $\rho$ numa outra configuração $\zeta$ na qual os spins +1 formam um único aglomerado. Se $M$ e $N$ são os lados do menor retângulo que engloba este aglomerado, temos: 


$$
\begin{aligned}
& H(\rho)-H(-1) \geq H(\zeta)-H(-\underline{1}) \geq \\
& 2(M+N)-h[L(L-1)+1]
\end{aligned}
$$

Como $\Gamma(h)=4 L-h[L(L-1)+1]$ obtemos

$$
H(\rho)-H(-1) \geq 2(M+N)-4 L+\Gamma(h)
$$

de forma que (2.28) segue se mostrarmos que $M+N \geq 2 L$. Seja $M=L+k$, para $k$ inteiro com $k>-L$. Para todo $k$ vale $k^{2}+k+1>0$, o que implica

$$
L-k<\frac{L(L-1)+1}{L+k}+1
$$

e portanto $L-k \leq\left\lceil\frac{L(L-1)+1}{L+k}\right\rceil$.

(p)"

Mas sé $M N \geq L(L-1)+1$ então $N \geq\left\lceil\frac{L(L-1)+1}{L+k}\right\rceil$ e, pela relação anterior, temos $N \geq L-k$ e portanto $M+N \geq 2 L$.

$\square_{\text {Lema } 12}$

Podemos agora passar a demostração do Lema 10.

De (2.18) e do fato de $\mathcal{P}_{i}$ ter que ser atravessado por $\left\{\sigma_{t}^{\eta_{i}}\right\}_{t \geq 0}$ para chegar a $E_{i}$ temos

$$
P\left(T^{\eta_{i}}\left(\mathcal{P}_{i}\right)<\widehat{T}^{\eta}\left(E_{i}\right)\right)=1
$$

onde $T^{\eta_{i}}\left(\mathcal{P}_{i}\right)=\inf \left\{t \geq 0: \sigma_{t}^{\eta_{i}} \in \mathcal{P}_{i}\right\}$

Defina o processo $\left\{\tilde{\sigma}_{t}^{\eta_{i}}\right\}_{t \geq 0}$ com configuração inicial $\eta_{i}$ e restrito a $\mathcal{H}_{i}$ acoplado a $\left\{\sigma_{t}^{\eta_{i}}\right\}_{t \geq 0}$ através do acoplamento $A$. Então

$$
P\left(\tilde{T}\left(\mathcal{P}_{i}\right)=T^{\eta_{i}}\left(\mathcal{P}_{i}\right)\right)=1
$$

onde $\tilde{T}\left(\mathcal{P}_{i}\right)=\inf \left\{t \geq 0: \tilde{\sigma}_{i}^{\eta_{i}} \in \mathcal{P}_{i}\right\}$ 
Pelos Lemas 2.4 e 2.5 podemos aplicar o Lema 2 obtendo que, se $\epsilon>0$

$$
\lim _{\beta \rightarrow \infty} P\left(\tilde{T}^{\eta_{i}}\left(\mathcal{P}_{i}<e^{\beta(\Gamma(h)-\varepsilon)}\right)=0\right.
$$

O Lema 10 segue de (2.34) junto com (2.33), (2.32) e (2.17).

$\square_{\text {Lema } 10}$

Dos Lemas 8 e 10 segue que $\left\{\hat{\sigma}_{t}^{\eta}\right\}_{t \geq 0}$ atinge, num tempo inferior a $e^{\beta(E(h)+\epsilon)}$, uma configuração $\rho \in \mathcal{M} \backslash\{\eta\} \operatorname{com} \rho \leq \eta$. Ocorre que só existe uma configuração $\rho \in$ $\mathcal{M} \backslash\{\eta\}$ com $\rho \leq \eta$ que possa ser atingida por esse processo que corresponde a configuração $\underline{\eta}$ com todos os spins -1 exceto aqueles em $\{1, \ldots, P\} \times\{1, \ldots, Q\} \times\{1, \ldots, R-1\}$.

Verifiquemos essa afirmação. Seja $\rho$ essa configuração em $\mathcal{M} \backslash\{\eta\}$ atingida pelo processo após deixar $\hat{B}(\eta)$. Todos os spins em $\{1, \ldots, P\} \times\{1, \ldots, Q\} \times\{1, \ldots, R-1\}$ são +1 ao longo de toda evolução do processo $\left\{\hat{\sigma}_{t}^{\eta}\right\}$ enquanto, pelo Lema 10 , a configuração $\rho$ nâo tem spins +1 fora de $\eta$. Se existem spins +1 na fatia $\{1, \ldots, P\} \times\{1, \ldots, Q\} \times\{R\}$ estes devem formar retângulos (caso contrário $\rho \notin M$ ). Se um desses retângulos tiver o menor lado inferior a $2 / h$ este tende a decrescer. Por outro lado se um deles tiver o menor lado maior que $2 / h$ e $\rho \neq \eta$ este retângulo tende a crescer. Como por hipótese $\rho \neq \eta$ a única solução é esta fatia não conter spins +1 .

Disso resulta que

$$
\lim _{\beta \rightarrow \infty} P\left(\hat{\sigma}_{\widehat{T}_{\eta}(\mathcal{M} \backslash\{\eta\})}^{\eta}=P\right)=1
$$

Esse resultado, junto com a limitação em $\hat{T}^{\eta}(\mu \mid\{\eta\})$ obtida do Lema 8 e a relação (2.2), implicam que o processo $\left\{\sigma_{t}^{\eta}\right\}_{t \geq 0}$ atinge uma configuração $\xi \leq \underline{\eta}$ num tempo inferior a $e^{\beta(E(h)+4 \epsilon)}$ com probabilidade que vai a 1 quando $\beta$ cresce. Se nesse instante recomeçarmos o processo na configuração $\eta \in \mathcal{R}$ podemos repetir o argumento de forma que o processo atinge -1 num tempo inferior a $e^{\beta(E(h)+5 \epsilon)}$. 


\section{Capítulo 3}

\section{Sistema bidimensional}

Este capítulo considera o sistema bidimensional. Provamos aqui os Teoremas 1 e 2 e em seguida apresentamos o esquema da prova do Teorema 3 . Esses teoremas, junto com outros resultados para o modelo de Ising estocástico bidimensional com dinâmica de Metrópolis são provados em [NS] através de uma abordagem diferente daquela que aqui apresentamos.

O Teorema 1 é, de fato, uma consequência imediata dos Lemas 1.5 e 1.7. No caso a) se $\eta \in \mathcal{R}$ e $l(\eta)=l<2 / h$ então $\delta_{\eta}=(l-1) h$ e da demonstração do Lema 1.5 segue que -1 é atingido num tempo inferior a $e^{\beta(l-1+c)}, \epsilon>0$, com probabilidade tendendo a 1 quando $\beta$ cresce. Quanto a parte b), se $\eta$ é tal que $l(\eta)>2 / h$, então $\delta_{\eta}=2-h$ e da demonstração do Lema 1.7 segue que $+\underline{1}$ é atingido num tempo inferior a $e^{\beta(2-h+\epsilon)}, \epsilon>0$, com probabilidade que vai a 1 quando $\beta$ cresce.

De fato no caso bidimensional temos $\mathcal{M}=\mathcal{M}_{1}=\{-\underline{1},+\underline{1}\}$.

Esse resultado permite separar o espaço de configuraçốes , $\Sigma$, em três subconjuntos disjuntos e não vazios $\mathcal{A}, \mathcal{B}$ e $\mathcal{C}$ tal que 
a) Se $\eta \in \mathcal{A}$ então

$$
\lim _{\beta \rightarrow \infty} P\left(T^{\eta}(-1)<T^{\eta}, T^{\eta}(-1)<\exp (\beta(2-h))\right)=1
$$

b) Se $\eta \in \mathcal{C}$ e $\epsilon>0$ então

$$
\lim _{\beta \rightarrow \infty} P\left(T^{\eta}<T^{\eta}(-\underline{1}), T^{\eta}<\exp (\beta(2-h+\epsilon))\right)=1
$$

c) Se $\eta \in \mathcal{B}$ então

$$
\begin{aligned}
& \liminf _{\beta \rightarrow \infty} P\left(T^{\eta}(-1)<T^{\eta}\right)>0 \\
& \liminf _{\beta \rightarrow \infty} P\left(T^{\eta}<T^{\eta}(-\underline{1})\right)>0
\end{aligned}
$$

e para todo $\epsilon>0$

$$
\lim _{\beta \rightarrow \infty} P\left(T^{\eta}(\{-1,+1\})<\exp (\beta(2-h+\epsilon))\right)=1
$$

As configurações de $\mathcal{A}$ são aquelas que pertencem apenas a cones de configurações $\eta \in M \operatorname{com} \delta_{\eta} \leq(L-2) h$ enquanto aquelas de $\mathcal{C}$ pertencem apenas a cones de configurações $\xi \in M \operatorname{com} \delta_{\xi} \geq 2-h$. As configurações que pertencem a cones de configurações dos dois tipos são as de $\mathcal{B}$

Demonstraremos agora o Teorema 2. Seja $\mathcal{D}=\mathcal{A} U \mathcal{B}$ e defina

$$
S=T-T(\mathcal{C})
$$

onde $T=T^{-1}(+\underline{1})$ e $T(\mathcal{C})=T^{-1}(\mathcal{C})$.

Começamos mostrando que

$$
\frac{T(\mathcal{C})}{\gamma_{\beta}} \rightarrow \tau \text { em distribuição quando } \beta \rightarrow \infty
$$

onde $\tau$ é uma variável aleatória com distribuição exponencial de média 1 e $\gamma_{\beta}$ é definido por

$$
P\left(T(\mathcal{C})>\gamma_{\beta}\right)=e^{-1}
$$


Em seguida mostramos que

$$
\frac{S}{\gamma_{\beta}} \rightarrow 0 \text { em probabilidade }
$$

de onde segue

$$
\frac{T}{\gamma_{\beta}} \rightarrow \tau \text { em distribuiçâo quando } \beta \rightarrow \infty \text {. }
$$

Em seguida verificamos que (3.2) implica no Teorema 2.

Para provar (3.2) consideramos o processo $\left\{\tilde{\sigma}_{t}^{\eta}\right\}_{t \geq 0}$, com configuração inicial $\eta \in \mathcal{D}$, restrito ao conjunto conexo $\mathcal{D}$ e acoplado a $\left\{\sigma_{t}^{\eta}\right\}_{t \geq 0}$ pelo acoplamento A.

Seja $\tilde{\mu}$ a medida invariante desse processo restrito a $\mathcal{D}$. O primeiro resultado importante é que $\tilde{\mu}$ concentra sua massa na configuração - 1 pois esse é o estado de menor energia em $\mathcal{D}$.

Lema 13 No caso fundamental em $d=2$

a) para todo $\eta \in \mathcal{D} \backslash\{-\underline{1}\}$ temos $H(\eta)>H(-\underline{1})$

b) $\tilde{\mu}$ concentra sua massa na configuração $-\underline{1}$ quando $\beta \rightarrow \infty$.

Dem : Se $\eta \in M$ então o Processo $X_{t}^{\eta}$ percorre configurações de M numa sequência com energias estritamente decrescentes até atingir $-\underline{1}$ ( isto segue dos argumentos da demonstração do Lema 5). Então $H(\eta)>H(-1)$ neste caso. Se $\eta \notin M$ então $\eta$ tem energia maior que toda configuração $\rho \in M \operatorname{com} \eta \in C(\rho)$ de forma que novamente segue $H(\eta)>H(-1)$. Parte b) é consequência imediata de a) pela definição de $\tilde{\mu} . \quad \square_{\text {Lema } 13}$

A demonstração de (3.2) é obtida mostrando que a variável aleatória $T(\mathcal{C})$, na escala dada por $\gamma_{\beta}$, tende a uma variável aleatória sem memória quando $\beta$ cresce. Mais precisamente, vamos mostrar que

$$
\lim _{\beta \rightarrow \infty} \Delta_{\beta}(s, t)=0
$$


onde

$$
\Delta_{\beta}(s, t)=\left|P\left(T(\mathcal{C})>(s+t) \gamma_{\beta}\right)-P\left(T(\mathcal{C})>s \gamma_{\beta}\right) P\left(T(\mathcal{C})>t \gamma_{\beta}\right)\right|
$$

Através do propriedade de Markov, temos

$$
P\left(T(\mathcal{C})>(s+t) \gamma_{\beta}\right)=\sum_{\eta \in \mathcal{D}} P\left(T(\mathcal{C})>s \gamma_{\beta}, \sigma_{s \gamma_{\beta}}^{-1}=\eta\right) P\left(T^{\eta}(\mathcal{C})>t \gamma_{\beta}\right)
$$

de forma que

$$
\Delta_{\beta}(s, t) \leq P\left(T(\mathcal{C})>s \gamma_{\beta}, \sigma_{s \gamma_{\beta}}^{-1} \neq-1\right) \leq P\left(\tilde{\sigma}_{s \gamma_{\beta}}^{-1} \neq-1\right)
$$

onde na última passagem usamos o processo $\left\{\tilde{\sigma}_{t}^{-1}\right\}_{t \geq 0}$ restrito a $\mathcal{D}$ definido anteriormente. Acoplamos agora esse processo, via acoplamento $\mathrm{B}, \operatorname{com}\left\{\tilde{\sigma}_{t}^{\tilde{\mu}}\right\}_{t \geq 0}$, também restrito a $\mathcal{D}$ e com configuração inicial escolhida conforme a medida $\tilde{\mu}$. Então

$$
P\left(\tilde{\sigma}_{s \gamma_{\beta}}^{-1} \neq-1\right) \leq P\left(\tilde{\sigma}_{0}^{-1} \neq \tilde{\sigma}_{0}^{\tilde{\mu}}\right)+P\left(\tilde{\sigma}_{s \gamma_{\beta}}^{\tilde{\mu}} \neq-1\right) \leq 2 \tilde{\mu}(\mathcal{D} \backslash\{-1\})
$$

onde usamos a invariância de $\tilde{\mu}$ na última desigualdade. Usando (3.7) em (3.6), o Lema 13 implica (3.5) e portanto (3.2).

Para provar (3.3) basta observar que $\delta_{-1}>4-h$ de forma que $\gamma_{\beta} \geq e^{\beta(4-h)}$ e que, pelo Lema 7 , o tempo de relaxação de configurações em $\mathcal{D}$ é menor que $e^{\beta(2-h+\epsilon)}$, $\epsilon>0$, com probabilidade que vai a 1 quando $\beta$ cresce.

Basta agora substituir $\gamma_{\beta}$ por $E T$ em (3.3). Por monotonicidade

$$
P\left(T>\gamma_{\beta} u\right) \leq\left(P\left(T>\gamma_{\beta}\right)\right)^{\lfloor u\rfloor}
$$

e de onde segue que

$$
\lim _{\beta \rightarrow \infty} \frac{E T}{\gamma_{\beta}}=\lim _{\beta \rightarrow \infty} \frac{\int_{0}^{\infty} P(T>t) d t}{\gamma_{\beta}}=\lim _{\beta \rightarrow \infty} \int_{0}^{\infty} P\left(T / \gamma_{\beta}>u\right) d u=\int_{0}^{\infty} e^{-u} d u=1
$$

terminando a demonstração do Teorema2. 
Apresentamos agora o esquema da prova do Teorema 3.

O primeiro passo consiste em obter uma limitação superior para $T$ mostrando que para todo $\epsilon>0$

$$
\lim _{\beta \rightarrow \infty} P(T>\exp (\beta(\Gamma(h)+3 \epsilon)))=0 .
$$

que corresponde ao análogo do Lema 8 e cuja demonstração é essencialmente a mesma. Começamos obtendo uma boa estimativa para a probabilidade do processo atingir $\mathcal{C}$ num tempo da ordem do tempo de relaxação em $\mathcal{D}$ a qual é conseguida verificando-se que para todo $\epsilon>0$ existe um $\beta_{0}<\infty$ tal que para $\beta>\beta_{0}$

$$
P\left(T(\mathcal{C}) \leq \exp (\beta((L-1) h+2 \epsilon)) \geq \frac{1}{3} \exp (-\beta e(h, L))\right.
$$

onde $e(h, L)=H(\eta)-H(-1)=4 L-L^{2} h$ para $\eta \in \mathcal{G}$. A desigualdade (3.10) implica (3.9) através de um argumento totalmente análogo ao apresentado em (2.14).

Seja $\mathcal{E}$ o conjunto das configurações nas quais todos os spins +1 estão dentro de um quadrado $L \times L$ e defina $\mathcal{F}=\mathcal{D} U \mathcal{E}$. Considere os processos $\left\{\tilde{\sigma}_{t}^{-1}\right\}_{t \geq 0}$ e $\left\{\tilde{\sigma}_{t}^{\tilde{\mu}}\right\}_{t \geq 0}$ restritos a $\mathcal{F}$ com configurações iniciais, respectivamente, -1 e escolhida com respeito a $\tilde{\mu}$, a medida de Gibbs restrita a $\mathcal{F}$. A relação (3.10) é consequência do fato das evoluções desses dois processos, com probabilidade que vai a 1 quando $\beta$ cresce, se tornarem indistinguíveis ao cabo de um tempo da ordem do tempo de relaxação em $\mathcal{D}$ e que, num dado instante, $\tilde{\sigma}_{t}^{\bar{\mu}}$ está em $\mathcal{F} \backslash \mathcal{D}$ com probabilidade $e^{-\beta e(h, L)}$.

Dessa limitação superior para $T$ obtemos parte c) do Teorema 3. Na passagem entre $-\underline{1}$ e $+\underline{1}$ o processo tem que atravessar o seguinte conjunto de configurações

$$
\mathcal{I}_{L^{2}}=\left\{\eta \in \Sigma:|\eta|=L^{2}\right\}
$$

uma vez que $|-1|=0$ e apenas um spin vira por vez na dinâmica do processo. Consideramos agora o processo $\left\{\sigma_{t}^{-1}\right\}_{t \geq 0}$ acoplado a $\left\{\tilde{\sigma}_{t}^{-1}\right\}_{t \geq 0}$ restrito ao conjunto conexo $\mathcal{H}$ através do acoplamento $B$ onde

$$
\mathcal{H}=\left\{\eta \in \Sigma:|\eta| \geq L^{2}\right\}
$$

A análise da energia das configurações em $\mathcal{I}_{L^{2}}$ mostra que somente aquelas no conjunto $\mathcal{G}$ têm energia inferior a $\Gamma(h)+3 \epsilon$ para $\epsilon>0$ suficientemente pequeno. Ao verificarmos que 
- 1 é o mínimo de energia em $\mathcal{H}$, o que é feito como no Lema 2, podemos aplicar o Lema 2 para concluir que, quando $\beta$ cresce, apenas as configurações de $\mathcal{G}$ dentre todas aquelas de $\mathcal{I}_{L^{2}}$ podem ser atingidas num tempo inferior a $e^{\beta(\Gamma(h)+3 \epsilon)}$ no qual, por $(3.9)$, o processo já saiu de $\mathcal{F}$ de forma que segue c).

Sabendo-se que o processo passou por $\mathcal{G}$ ao deixar $\mathcal{D}$ pela primeira vez uma análise relativamente simples de como $\mathcal{G}$ pode ser atingido a partir de configurações com magnetização menor implica que, com grande probabilidade para $\beta$ grande, esse conjunto foi atingido após o processo passar por $\mathcal{P}$ de forma que segue $\mathrm{d}$ ). Do fato de $\mathcal{P}$ ter sido atingido na passagem de $-\underline{1}$ para $+\underline{1}$ segue que o tempo gasto nesta passagem, com probabilidade que vai a 1 quando $\beta$ cresce, deve ser maior que $e^{\beta(\Gamma(h)-\epsilon)}, \epsilon>0$, uma vez que $\Gamma(h)$ é a altura da barreira de energia representada por $\mathcal{P}$. Essa limitação superior para $T$, junto com (3.9), termina a demonstração de a). 


\section{Referências}

[Bra]- S.Brassesco: Tunelling for a non-linear heat equation with noise. Preprint (1989).

[CGOV]- M.Cassandro, A.Galves, E.Olivieri, M.E.Vares: Metastable behavior of stochastic dynamics: A pathwise approach. J. Stat. Phys. 35 (1984) 603-634.

[COP]- M.Cassandro, E.Olivieri, P.Picco: Small random perturbations of infinite dimensional dynamical systems and nucleation theory. Ann. Inst. Henri Poincaré (Phys. Theor.) 44 (1986) 343-396.

[GOV]- A.Galves, E.Olivieri, M.E.Vares: Metastability for a class of dynamical systems subject to small random perturbations. Ann. Probab. 15 (1987) 1288-1305.

[EGJL]- V.R.Eston, A.Galves, C.M.Jacobi, R.Langevin: Dominance switch between two interacting species and metastability. Preprint (1988).

[Gla]- R.J. Glauber: Time Dependent Statistics of the Ising Model. J. Stat. Phys. 4294 (1963).

[KN]- C.Kipnis, C.M.Newman: The metastable behavior of infrequently observed, weakly random, one dimensional diffusion processes. SIAM J. Appl. Math. 45 (1985) 972-982.

[Lig]- T.M.Liggett: Interacting Particle Systems. Springer, 1985.

[LS]- J.L.Lebowitz, R.H.Schonmann: On the asymptotics of occurence times of rare events for stochastic spin systems. J. Stat. Phys. 48 (1987) 727-751.

[MOS]- F.Martinelli, E.Olivieri, E.Scoppola: Small random perturbation of finite and 
infinite dimensional systems: unpredictability of exit times. Preprint (1988).

[NCK]- C.M.Newman, J.E.Cohen, C.Kipnis: Neo-darwinian evolution implies punctuated equilibria. Nature 315 (1985) 400-401.

[N]- E. Jordão Neves: Some Remarks on Schonmann-Tanaka's One Dimensional Caricature of Phase Transition: em preparação.

[NS]- E.Jordão Neves, Roberto H. Schonmann: Critical Droplets and Metastability for a Glauber Dynamics at Very Low Temperatures. Submetido ao Comm. Math. Phys. (1989).

[Sch]- R.H.Schonmann: Metastability for the contact process. J. Stat. Phys. 41 (1985) 445-464.

[ST]- R.H.Schonmann, N.I. Tanaka: One Dimensional Caricature of Phase Transition; Preprint USP (1990). 